\title{
A family of lower- and higher-order transversal linearization techniques in non-linear stochastic engineering dynamics
}

\author{
D. $\operatorname{Roy}^{*, \dagger} \dagger$ \\ Structures Laboratory, Department of Civil Engineering, Indian Institute of Science, Bangalore 560012 , India
}

\begin{abstract}
SUMMARY
Sample pathwise numerical integration of noise-driven engineering dynamical systems cannot generally be performed beyond a limited level of accuracy, especially when the noise processes are modelled using (filtered) white noises. Recently, a locally transversal linearization (LTL) strategy has been proposed by the author (Proc Roy Soc London A 2001; 457:539-566) for direct integration of deterministic and stochastic non-linear dynamical systems. The present effort is focussed on a host of extensions along with detailed theoretical error analyses of the linearization approach, especially as applied to problems in non-linear stochastic engineering dynamics. Thus, to begin with, estimates of local and global error orders in the basic LTL scheme are obtained separately for the displacement and velocity vectors when the system is driven either by a set of additive noises or by an arbitrary combination of (independently evolving) additive and multiplicative noises. Following this, a new family of higher-order LTL schemes is proposed in order to improve upon the basic LTL method and the associated error orders are established. A stepwise implementation of the lower- and higher-order versions of the LTL method, along with certain computational aspects, is also outlined. The proposed schemes are numerically illustrated, to a limited extent, for a single degree-of-freedom (SDOF) and a two degree-of-freedom (TDOF) non-linear engineering systems under additive and/or multiplicative white noise excitations. Copyright (c) 2004 John Wiley \& Sons, Ltd.
\end{abstract}

KEY WORDS: transversal linearization; higher-order schemes; Ito-Taylor expansions; local and global error orders; engineering systems; additive and multiplicative noises

\section{THE INTRODUCTION}

Accurate pathwise integration of non-linear stochastic dynamical systems under additive or multiplicative white noise inputs (i.e. formal derivatives of Wiener processes) poses several challenges to scientists and engineers. Since Wiener processes are non-differentiable [1], a white noise process is not a valid mathematical function of time. Thus, taking for an instance an engineering dynamical system driven by white noise(s), the acceleration vector does not

\footnotetext{
${ }^{*}$ Correspondence to: D. Roy, Structures Laboratory, Department of Civil Engineering, Indian Institute of Science, Bangalore 560 012, India.

†E-mail: royd@civil.iisc.ernet.in

${ }^{\ddagger}$ Associate Professor
}

Published online 19 August 2004

Received 11 October 2002

Copyright (c) 2004 John Wiley \& Sons, Ltd.

Revised 5 September 2003

Accepted 19 December 2003 
have any mathematical meaning. To further complicate matters, Wiener increments, unlike a deterministic functional increment, change by $O\left(h^{1 / 2}\right)$ in a time interval of $h$. It is thus natural that integration techniques for stochastic differential equation (SDE) should be so different from their deterministic counterparts [2]. The stochastic Taylor expansion [3] provides a way to generate consistently higher-order numerical integration schemes. The stochastic Euler scheme, essentially a stochastic Taylor expansion of upto $O(h)$ terms, has $O(h)$ and $O\left(h^{1 / 2}\right)$ local and global errors of convergence, respectively [4-6]. By including more terms in the expansion, strong Taylor schemes of $O\left(h^{3 / 2}\right)$ and $O\left(h^{2}\right)$ have been proposed, among others, by Wagner and Platen [7], Milstein [8] and Kloeden and Platen [9]. The book by Kloeden and Platen [3] has a fairly extensive review of all these methods. However, the major downside in applying these schemes is the enormous difficulties in evaluating multiple stochastic integrals. An additional difficulty is to evaluate the derivatives of drift and diffusion vectors. One may alternatively use stochastic Runge-Kutta type schemes [10]. For SDEs with a one-dimensional Wiener process, the stochastic Heun scheme (SHS), which is essentially a stochastic version of the deterministic trapezoidal rule, appears to be the most general as well as accurate integration scheme of strong (local) order 1.5 [2]. However, for SDEs with a higher-dimensional Wiener process vector, SHS generally only yields a local error order 1.0 unless certain conditions on the gradients of the diffusion terms are met with. In fact, Rumelin [10] has demonstrated that, following the Runge-Kutta route, one can derive methods of higher order of accuracy than SHS if and only if certain very restrictive equalities involving the partial derivatives of the drift and diffusion coefficients are satisfied.

Quite recently, a semi-analytical integration method, called the locally transversal linearization (LTL), has been proposed by the author [11] for non-linear stochastic dynamical systems. The essence of the method is to locally construct a set of conditionally linear and easily integrable (non-unique) system of SDEs such that, with known initial conditions at the left end of a given time interval, solution vector of the linearized SDE transversally intersects that of the original SDE at the right end of the same interval. Even though the LTL method appears to have several advantages over most existing schemes, estimates of local and global error orders during integration are presently not known. Moreover, the possibility of improving the accuracy of integration by constructing higher-order LTL systems remains unexplored. A purpose of this study is to adequately fill up these voids and also to extend these new developments to sufficiently general engineering dynamical systems. Towards this, local and global error estimates for the displacement and velocity vectors, as obtained via the lower-order LTL scheme (also referred to as the basic LTL scheme) are provided. A method for constructing higher-order LTL schemes, whereby orders of accuracy in computing the response vectors are increased, is next dealt with in detail. Both additive and multiplicative noise excitations are included in the analyses. No restrictions are imposed as to the number of white noise inputs to the system. A few numerical illustrations for the basic and higher-order LTL schemes are provided, first for a single-degree-of-freedom (SDOF) Duffing problem and then a two-degree-of-freedom (TDOF) symmetrical non-linear problem.

\section{THE LOWER ORDER OR BASIC LTL METHOD}

Consider a very general $N$-DOF non-linear stochastic engineering dynamical system in the following canonical form: 


$$
\ddot{X}=A(X, \dot{X}, t)+\sum_{r=1}^{q} B_{r}(X, \dot{X}, t) \dot{W}_{r}(t)
$$

where $X, \dot{X} \in \mathfrak{R}^{n}, A(X, \dot{X}, t): \mathfrak{R}^{N} \times \mathfrak{R}^{N} \times \mathfrak{R} \rightarrow \mathfrak{R}^{N}$ is an $N$-dimensional drift vector function (non-linear in $X$ and $\dot{X}$ and with or without explicit time dependence), every element $b_{r}^{(j)}(X, \dot{X}, t)$ of the set $\left\{B_{r}(X, \dot{X}, t) \mid r=1,2, \ldots, q\right\}$ is an $N$-dimensional diffusion vector, and $\left\{W_{r}(t) \mid r=1,2, \ldots, q\right\}$ denotes a set of independently evolving Wiener processes with $W_{r}(0)=0$ and $E\left[\left|W_{r}(t)-W_{r}(s)\right|^{2}\right]=(t-s), t>s$, so that $\left\{\dot{W}_{r}(t) \mid r=1,2, \ldots, q\right\}$ constitutes the corresponding set of the so-called 'white noise processes'. It may be noted that the overdot (meaning differentiation w.r.t time, $t$ ) over $W_{r}(t)$ needs to be construed in a formal sense since Wiener processes are only continuous and not differentiable in $t$. From this point of view, the acceleration vector $\ddot{X}$ also does not make much of mathematical sense and thus it would be more preferable to cast the system of second-order Equations (1) via the following incremental form in the state space:

$$
\begin{aligned}
& \mathrm{d} X_{1}=X_{2} \mathrm{~d} t \\
& \mathrm{~d} X_{2}=A\left(X_{1}, X_{2}, t\right) \mathrm{d} t+\sum_{r=1}^{q} B_{r}\left(X_{1}, X_{2}, t\right) \mathrm{d} W_{r}(t)
\end{aligned}
$$

where $X_{1}=\left\{x_{1}^{(1)}, x_{1}^{(2)}, \ldots, x_{1}^{(N)}\right\}^{\mathrm{T}}$ and $X_{2}=\left\{x_{2}^{(1)}, x_{2}^{(2)}, \ldots, x_{2}^{(N)}\right\}^{\mathrm{T}}$ are, respectively, the displacement and velocity vector components of the $2 N$-dimensional response vector $X=\left\{X_{1}^{\mathrm{T}}\right.$, $\left.X_{2}^{\mathrm{T}}\right\}^{\mathrm{T}}$. Similarly, the vectors $A$ and $B_{r}$ may be written in terms of their scalar components as $A=\left\{a^{(j)} \mid j=1,2, \ldots, N\right\}$ and $B_{r}=\left\{b_{r}^{(j)} \mid j=1,2, \ldots, N\right\}$. It is assumed that these vector drift and diffusion functions are measurable with respect to all the arguments, Lipschitz continuous and have appropriate growth bounds (not necessarily linear). Thus the sample continuity of any realization of the (separable) non-linear flow $\phi_{t}\left(\omega, X_{1}(0), X_{2}(0)\right)$ for any $\omega \in \Omega(\Omega$ being the event space) is assured provided that the (norms of) initial displacement and velocity vectors, $X_{1}(0), X_{2}(0) \in R^{N}$, assumed to be deterministic without the loss of any generality, are bounded. In all the discussion to follow, no differentiability requirements are imposed on the drift and diffusion vectors, unless specifically mentioned on the contrary. However, it is assumed that the drift vector is separable in the way as described below

$$
A\left(X_{1}, X_{2}, t\right)=A_{1}\left(X_{1}, X_{2}\right)+f_{\mathrm{e}}(t)+A_{\mathrm{n}}\left(X_{1}, X_{2}, t\right)
$$

where

$$
\left\{A_{1}\left(X_{1}, X_{2}\right)\right\}=\left[C_{1}\right]\left\{X_{2}\right\}+\left[K_{1}\right]\left\{X_{1}\right\}
$$

and

$$
\left\{A_{\mathrm{n}}\left(X_{1}, X_{2}, t\right)\right\}=\left[C_{\mathrm{n}}\left(X_{1}, X_{2}, t\right)\right]\left\{X_{2}\right\}+\left[K_{\mathrm{n}}\left(X_{1}, X_{2}, t\right)\right]\left\{X_{1}\right\}
$$

In the above equations, $f_{\mathrm{e}}(t)=\left\{f_{\mathrm{e}}^{(j)}(t) \mid j=1,2, \ldots, N\right\}$ is the non-parametric, external force vector, $A_{1}=\left\{a_{1}^{(j)} \mid j=1, \ldots, N\right\}$ is the linear, time-invariant part of the drift vector and is decomposable in terms of the constant damping and stiffness matrices, denoted respectively, as $C_{1}$ and $K_{1}$ in Equation (3b). Similarly, the parametric and/or non-linear part of the vector field, denoted by $A_{\mathrm{n}}\left(X_{1}, X_{2}, t\right)=\left\{a_{\mathrm{n}}^{(j)}\left(X_{1}, X_{2}, t\right)\right\}$, is assumed to be decomposable as indicated 
in Equation (3c), where $C_{\mathrm{n}}$ and $K_{\mathrm{n}}$, respectively, stand for the time and state-dependent, conditionally known damping and stiffness matrices, respectively. Finally, the diffusion vector, $B_{r}\left(X_{1}, X_{2}, t\right)$, is assumed to be separable in terms of its additive (possibly time-dependent) and multiplicative constituents as

$$
B_{r}\left(X_{1}, X_{2}, t\right)=\sigma_{r}(t)+\mu_{r}\left(X_{1}, X_{2}, t\right), \quad r=1,2, \ldots, q
$$

Let the subset of the time axis over $[0, T]$, which is the interval over which integration needs to be performed, be ordered such that $0=t_{0}<t_{1}<t_{2}<\cdots<t_{i}<\cdots<t_{P}=T$ and $h_{i}=t_{i}-t_{i-1}$ where $i \in \mathrm{Z}^{+}$. The purpose of the LTL method is to replace the non-linear system of SDEs (2) by a suitably chosen set of $P$ linear systems of SDEs, wherein the $i$ th linear system should, in a sense, be a representative of the non-linear flow over the $i$ th time interval $T_{i}=\left(t_{i-1}, t_{i}\right]$. Such a replacement is non-unique [11]; however the following system of conditionally linearized SDEs constitutes a valid LTL system corresponding to Equation (2) over the interval $T_{i}$ :

$$
\begin{aligned}
\mathrm{d} \bar{X}_{1}= & \bar{X}_{2} \mathrm{~d} t \\
\mathrm{~d} \bar{X}_{2}= & {\left[A_{1}\left(\bar{X}_{1}, \bar{X}_{2}\right)+f_{\mathrm{e}}(t)+C_{\mathrm{n}}\left(X_{1, i}, X_{2, i}, t_{i}\right) \bar{X}_{2}+K_{\mathrm{n}}\left(X_{1, i}, X_{2, i}, t_{i}\right) \bar{X}_{1}\right] \mathrm{d} t } \\
& +\sum_{r=1}^{q}\left[\sigma_{r}(t)+\mu_{r}\left(X_{1, i}, X_{2, i}, t\right)\right] \mathrm{d} W_{r}(t)
\end{aligned}
$$

In the above equation, $X_{1, i} \triangleq X_{1}\left(t_{i}\right), X_{2, i} \triangleq X_{2}\left(t_{i}\right)$ and the linearized system has to be solved subject to the known initial condition vector $\left(X_{1, i-1}, X_{2, i-1}\right) \in \mathfrak{R}^{2 N}$. It may be readily observed that the vector fields of the non-linear and transversally linearized equations (i.e. the right-hand sides of Equations (2) and (5) respectively) are instantaneously identical at $t=t_{i}$ (and not away from $t=t_{i}$ ) provided one can enforce the following vector identities:

$$
X_{1, i}=\bar{X}_{1, i} ; \quad X_{2, i}=\bar{X}_{2, i}
$$

Denoting by $u_{i}=\left(X_{1, i}, X_{2, i}, t_{i}\right)$ and $\bar{u}_{i}=\left(\bar{X}_{1, i}, \bar{X}_{2, i}, t_{i}\right)$, the state-space discretized solutions (i.e. discrete points in the associated phase spaces) to the non-linear and linearized SDEs at $t=t_{i}$, one may readily observe (also see Reference [11] for further details) that the corresponding tangent spaces $T_{u i}$ and $T_{\bar{u} i}$ (constructed, respectively, at $u_{i}$ and $\bar{u}_{i}$ ) are transversal (non-tangential). It is known that the local evolutions of solutions $\Phi_{t}\left(\omega, X_{1, i-1}, X_{2, i-1}\right), \bar{\Phi}_{t}(\omega$, $\left.X_{1, i-1}, X_{2, i-1}\right)$ for $t \in\left(t_{i-1}, t_{i}\right]$ and $\omega \in \Omega$ fixed, of the non-linear and linearized SDEs, respectively, are governed by their respective tangent spaces. One may thus argue that $\Phi_{t} \& \bar{\Phi}_{t}$ are transversal to each other on and around $t=t_{i}$. Moreover, if Equations (6) are somehow satisfied, so that $u_{i}=\bar{u}_{i}$, then it implies that the two locally transversal solution manifolds (which are locally homeomorphic to $\Phi_{t}\left(\omega, X_{1, i-1}, X_{2, i-1}\right)$ and $\left.\bar{\Phi}_{t}\left(\omega, X_{1, i-1}, X_{2, i-1}\right)\right)$ intersect at $t=t_{i}$. In other words, the discretized unknown vector $\left(X_{1, i}, X_{2, i}\right)$ may be obtained as the point of transversal intersections of $\Phi_{t}\left(\omega, X_{1, i-1}, X_{2, i-1}\right)$ and $\bar{\Phi}_{t}\left(\omega, X_{1, i-1}, X_{2, i-1}\right)$ at $t=t_{i}$. Since the point of intersection is common to non-linear and linearized manifolds, it should be possible to arrive at the point based on the solutions of the linearized SDEs (5). Indeed, a closed form solution, $\bar{X}(t)=\left\{\bar{X}_{1}^{\mathrm{T}}(t), \bar{X}_{2}^{\mathrm{T}}(t)\right\}^{\mathrm{T}}$, for Equation (5) may be conditionally constructed in terms 
of the unknown state vector, $X_{i}=\left\{X_{1, i}, X_{2, i}\right\}^{\mathrm{T}}$, as

$$
\bar{X}\left(t_{i}\right)=\left[\Phi\left(X_{i}, t_{i}, t_{i-1}\right)\right]\left\{\begin{array}{c}
X_{i-1}+\int_{t_{i-1}}^{t_{i}}\left[\Phi^{-1}\left(X_{i}, s, t_{i-1}\right)\right] \hat{f}(s) \mathrm{d} s \\
\quad+\int_{t_{i-1}}^{t_{i}}\left[\Phi^{-1}\left(X_{i}, s, t_{i-1}\right)\right] \sum_{r=1}^{q}\left(\hat{\sigma}_{r}(s)+\hat{\mu}\left(X_{i}, s\right)\right) \mathrm{d} W_{r}(s)
\end{array}\right\}
$$

In the above expression, $\hat{\sigma}_{r}(s)=\left\{\{0\}^{\mathrm{T}},\left\{\sigma_{r}\right\}^{\mathrm{T}}\right\}^{\mathrm{T}}$ is the $2 N$-dimensional additive diffusion vector, obtained by pre-augmenting $\sigma_{r}$ with an $N$-dimensional zero vector, $\{0\}, \hat{\mu}_{r}\left(X_{i}, s\right)=$ $\left\{\{0\}^{\mathrm{T}},\left\{\mu_{r}\left(X_{i}, s\right)\right\}^{\mathrm{T}}\right\}^{\mathrm{T}}$ is the $2 N$-dimensional, conditionally additive version of the originally multiplicative diffusion vector and $\hat{f}(s)=\left\{\{0\}^{\mathrm{T}},\left\{f_{\mathrm{e}}(s)\right\}^{\mathrm{T}}\right\}^{\mathrm{T}}$ is the $2 N$-dimensional deterministic force vector. Moreover, $\Phi\left(X_{i}, t, t_{i-1}\right)$ is a conditionally defined fundamental solution matrix (FSM) and has the following simple form:

$$
\Phi\left(x_{i}, t, t_{i-1}\right)=\exp \left\{\left[M\left(X_{i}, t_{i}\right)\right]\left(t-t_{i-1}\right)\right\}
$$

where the $2 N \times 2 N$ coefficient matrix, $M$, corresponding to the transversally linearized system, has the form

$$
[M]=\left[\begin{array}{cc}
{[0]} & {[I]} \\
{\left[K_{1}\right]+\left[K_{\mathrm{n}}\left(X_{i}, t_{i}\right)\right]} & {\left[C_{1}\right]+\left[C_{\mathrm{n}}\left(X_{i}, t_{i}\right)\right]}
\end{array}\right]
$$

Details of evaluating the matrix exponent, as needed to obtain the RHS of Equation (8), will be considered later. The linearized solution, provided in Equation (7), is conditional as it is expressible only in terms of the unknown, discretized solution vector $X_{i}$. At this stage, $X_{i}=\left(X_{1, i}^{\mathrm{T}}, X_{2, i}^{\mathrm{T}}\right)^{\mathrm{T}}$ may be determined by substituting the linearized solution (7) for $\bar{X}_{i}=$ $\left(\bar{X}_{1, i}^{\mathrm{T}}, \bar{X}_{2, i}^{\mathrm{T}}\right)^{\mathrm{T}}$ on the right-hand sides of the constraint (or, intersection) condition (6). Such an exercise readily results in a system of $2 N$ (coupled) non-linear algebraic equations for as many unknowns to determine the $2 N$-dimensional vector variable $X_{i}$. Roots (not necessarily unique) of these algebraic equations may be found via a Newton-Raphson search algorithm. A possible multiplicity of roots is consistent with the fact that non-linear dynamical systems may undergo bifurcations and thus may have multiple solutions.

\subsection{Error estimates}

The sample path, $X(t)$ traced by Equation (2) is, in general, different from the approximated LTL solution, $\bar{X}$ corresponding to Equation (5), unless the trajectory is in a phase-independent regime (see Reference [11] for details). Thus in the more common case of phase-dependent solutions (these include transient solutions), an instantaneous error at $t=t_{i}$ may be defined as the $2 N$-dimensional vector $E_{i}=\left\{E_{1, i}^{(j)} E_{2, i}^{(j)}\right\}=\left\{\left(x_{1, i}^{(j)}-\bar{x}_{1, i}^{(j)}\right),\left(x_{2, i}^{(j)}-\bar{x}_{2, i}^{(j)}\right)\right\}$, where $j=1, \ldots, N$, and the instantaneous Euclidean error norm is denoted as $e_{i}=\left\|X_{i}-\bar{X}_{i}\right\|$. The error vector may be treated as a set of conditional random variables such that the local initial condition, $X_{i-1}$, is deterministic and that $\bar{X}_{i-1}=X_{i-1}$. Let $r_{\mathrm{m}}$ and $r_{\mathrm{s}}$, respectively, denote the orders of the mean and mean square of the conditional (local) error with respect to the uniformly chosen 
time step size, $h=t_{i}-t_{i-1}$. Then, one can define the following local error bounds:

$$
\begin{aligned}
\left\|E\left(X_{i}-\bar{X}_{i}\right)\right\| & \leqslant Q\left(1+\left\|X_{i-1}\right\|^{2}\right) h^{r_{\mathrm{m}}} \\
{\left[E\left\|X_{i}-\bar{X}_{i}\right\|^{2}\right]^{1 / 2} } & \leqslant Q\left(1+\left\|X_{i-1}\right\|^{2}\right) h^{r_{\mathrm{s}}}
\end{aligned}
$$

\section{Proposition 1}

Let $r_{\mathrm{s}} \geqslant \frac{1}{2}$ and $r_{\mathrm{m}} \geqslant r_{\mathrm{s}}+\frac{1}{2}$. Then, one has the following bound on the global error:

$$
\left[E\left\|X_{i}-\bar{X}_{i}\right\|^{2}\right]^{1 / 2} \leqslant Q\left(1+\left\|X_{0}\right\|^{2}\right) h^{r_{\mathrm{s}}-1 / 2}
$$

Put another way, the global order of accuracy of the method, constructed using a one-step approximation, is $r_{\mathrm{g}}=r_{\mathrm{s}}-\frac{1}{2}$.

Proof

See the monograph by Milstein [8, pp. 12-17] for a step-by-step proof of this important proposition.

Presently, the error estimates would be performed based on stochastic Ito-Taylor expansions of the non-linear and conditionally linear vector fields. These expansions are in turn derived based on a repeated application of Ito's formula [5], which, as adapted specifically for Equation (2), is stated below:

$$
\begin{aligned}
f\left(X_{1}(s), X_{2}(s), s\right)= & f\left(X_{1}\left(t_{i-1}\right), X_{2}\left(t_{i-1}\right), t_{i-1}\right)+\sum_{r=1}^{q} \int_{t_{i-1}}^{s} \Lambda_{r} f\left(X_{1}\left(s_{1}\right), X_{2}\left(s_{1}\right), s_{1}\right) \mathrm{d} W_{r}\left(s_{1}\right) \\
& +\int_{t_{i-1}}^{s} L f\left(X_{1}\left(s_{1}\right), X_{2}\left(s_{1}\right), s_{1}\right) \mathrm{d} s_{1}
\end{aligned}
$$

where $f$ is any sufficiently differentiable (scalar or vector) function of its arguments, $s \geqslant t_{i-1}$ and the operators $\Lambda_{r}$ and $L$ are defined through their actions on $f$ as

$$
\begin{aligned}
& \Lambda_{r} f=\sum_{j=1}^{N} b_{r}^{(j)} \frac{\partial f\left(X_{1}, X_{2}, t\right)}{\partial x_{2}^{(j)}} \\
& L f=\frac{\partial f}{\partial t}+\sum_{j=1}^{N} x_{2}^{(j)} \frac{\partial f}{\partial x_{1}^{(j)}}+\sum_{j=1}^{N} a^{(j)} \frac{\partial f}{\partial x_{2}^{(j)}}+0.5 \sum_{r=1}^{q} \sum_{k=1}^{N} \sum_{l=1}^{N} b_{r}^{(k)} b_{r}^{(l)} \frac{\partial^{2} f}{\partial x_{2}^{(k)} \partial x_{2}^{(l)}}
\end{aligned}
$$

For convenience of further discussion, it is also necessary to define a multiple stochastic integral as

$$
I_{j_{1}, j_{2}, \ldots, j_{k}}=\int_{t_{i-1}}^{t_{i}} \mathrm{~d} W_{j_{k}}(s) \int_{t_{i-1}}^{s} \mathrm{~d} W_{j_{k-1}}\left(s_{1}\right) \int_{t_{i-1}}^{s_{1}} \cdots \int_{t_{i-1}}^{s_{k-2}} \mathrm{~d} W_{j_{1}}\left(s_{k-1}\right)
$$


where the integers $j_{1}, j_{2}, \ldots, j_{k}$ take values in the set $\{0,1,2, \ldots, q\}$ and $I_{j_{1}, j_{2}, \ldots, j_{k}}$ is called the $k$ th Ito multiple integral. Moreover, $\mathrm{d} W_{0}(s)$ is taken to indicate $\mathrm{d} s$. At this stage, the following proposition is essential.

\section{Proposition 2}

One has $E\left(I_{j_{1}, j_{2}, \ldots, j_{k}}\right)=0$ if there exists at least one $j_{m} \neq 0, m=1,2, \ldots, k$. On the other hand, $E\left(I_{j_{1}, j_{2}, \ldots, j_{k}}\right)=O\left(h^{k}\right)$ if $j_{m}=0 \forall m \in[0, k]$. Additionally, the following identity holds:

$$
\left.E\left(I_{j_{1}, j_{2}}, \ldots, j_{k}\right)^{2}\right]^{1 / 2}=O\left(h^{w}\right)
$$

where

$$
w=\sum_{m=1}^{k}\left(2-\bar{j}_{m}\right) / 2, \quad \bar{j}_{m}=1 \quad \text { if } j_{m} \neq 0, \text { else } \bar{j}_{m}=0
$$

Proof

The first part of the above proposition regarding the mean is quite straightforward. For the second part, involving Equation (15), reference is made to the monographs by Milstein [8] or Kloeden and Platen [3].

2.1.1. The case of only additive noises. To begin with, the case of purely additive stochastic excitations (possibly with time dependent coefficients), i.e. from Equation (4), $B_{r}(X, t)=$ $\left\{\sigma_{r}^{(j)}(t) \mid j=1, \ldots, N\right\}$, is considered. Using the original vector field as in Equation (2), the displacement components, $x_{1}^{(j)}, j=1,2, \ldots, N$, may be expanded in a stochastic Taylor series, which is obtainable through repeated applications of Ito's formula given by Equation (13). Thus, referring to Equation (13) with $f\left(X_{1}, X_{2}, t\right)=x_{1}^{(j)}(t)$, one readily has

$$
x_{1, i}^{(j)}=x_{1, i-1}^{(j)}+\int_{t_{i-1}}^{t_{i}} x_{2}^{(j)}(s) \mathrm{d} s
$$

Now, one may use $f\left(X_{1}, X_{2}, s\right)=x_{2}^{(j)}(s)$ and apply Ito's formula once more to arrive at

$$
\begin{aligned}
x_{1, i}^{(j)}= & x_{1, i-1}^{(j)}+x_{2, i-1}^{(j)} h+\sum_{r=1}^{q} \int_{t_{i-1}}^{t_{i}} \int_{t_{i-1}}^{s} \sigma_{r}^{(j)}\left(s_{1}\right) \mathrm{d} W_{r}\left(s_{1}\right) \mathrm{d} s \\
& +\int_{t_{i-1}}^{t_{i}} \int_{t_{i-1}}^{s} a^{(j)}\left(X_{1}\left(s_{1}\right), X_{2}\left(s_{1}\right), s_{1}\right) \mathrm{d} s_{1} \mathrm{~d} s
\end{aligned}
$$

The Ito's formula may again be applied with $f\left(X_{1}, X_{2}, s\right)=\sigma_{r}^{(j)}(s)$ and $f\left(X_{1}, X_{2}, s\right)=$ $a^{(j)}\left(X_{1}(s), X_{2}(s), s\right)$ to expand the two integrands associated with the two double integrals in Equation (16b). This results in

$$
x_{1, i}^{(j)}=x_{1, i-1}^{(j)}+x_{2, i-1}^{(j)} h+\sum_{r=1}^{q} \sigma_{r}^{(j)}\left(t_{i-1}\right) I_{r 0}+a^{(j)}\left(X_{1, i-1}, X_{2, i-1}, t_{i-1}\right) \frac{h^{2}}{2}+T_{1}^{(j)}
$$


where, $a^{(j)}$ denotes the $j$ th component of the velocity drift vector $A$, and the remainder is given by

$$
\begin{aligned}
T_{1}^{(j)}= & \sum_{r=1}^{q} \int_{t_{i-1}}^{t_{i}} \int_{t_{i-1}}^{s} \int_{t_{i-1}}^{s_{1}} \Lambda_{r} a^{(j)}\left(X\left(s_{2}\right), s_{2}\right) \mathrm{d} W_{r}\left(s_{2}\right) \mathrm{d} s_{1} \mathrm{~d} s \\
& +\int_{t_{i-1}}^{t_{i}} \int_{t_{i-1}}^{s} \int_{t_{i-1}}^{s_{1}} L a^{(j)}\left(X\left(s_{2}\right), s_{2}\right) \mathrm{d} s_{2} \mathrm{~d} s_{1} \mathrm{~d} s \\
& -\int_{t_{i-1}}^{t_{i}} \int_{t_{i-1}}^{s} \int_{t_{i-1}}^{s_{1}} \dot{\sigma}_{r}^{(j)}\left(s_{2}\right) \mathrm{d} s_{2} \mathrm{~d} W_{r}\left(s_{1}\right) \mathrm{d} s
\end{aligned}
$$

An implicit form of Equation (16c) may be written by noting that

$$
a^{(j)}\left(X_{i-1}, t_{i-1}\right)=a^{(j)}\left(X_{i}, t_{i}\right)-\sum_{r=1}^{q} \int_{t_{i-1}}^{t_{i}} \Lambda_{r} a^{(j)}(X(s), s) \mathrm{d} s-\int_{t_{i-1}}^{t_{i}} L a^{(j)}(X(s), s) \mathrm{d} s
$$

Thus Equation (16c) takes the form

$$
x_{1, i}^{(j)}=x_{1, i-1}^{(j)}+x_{2, i-1}^{(j)} h+\sum_{r=1}^{q} \sigma_{r}^{(j)}\left(t_{i-1}\right) I_{r 0}+a^{(j)}\left(X_{i}, t_{i}\right) h^{2} / 2+R_{1}^{(j)}
$$

The displacement remainder, corresponding to the final form of the displacement map governed by Equation (19), is given by

$$
R_{1}^{(j)}=T_{1}^{(j)}-\left(\sum_{r=1}^{q} \int_{t_{i-1}}^{t_{i}} \Lambda_{r} a^{(j)}(X, s) \mathrm{d} W_{r}(s)+\int_{t_{i-1}}^{t_{i}} L a^{(j)}(X, s) \mathrm{d} s\right) h^{2} / 2
$$

A similar expansion for the LTL-based displacement component, $\bar{x}_{1}^{(j)}(j=1,2, \ldots, N)$, subject to same initial conditions, $\left(X_{1, i-1}, X_{2, i-1}\right)$, leads to

$$
\begin{aligned}
\bar{x}_{1, i}^{(j)}= & x_{1, i-1}^{(j)}+x_{2, i-1}^{(j)} h+\sum_{r=1}^{q} \sigma_{r}^{(j)}\left(t_{i-1}\right) I_{r 0}+\left\{\left[C_{1}^{(j)}+C_{\mathrm{n}}^{(j)}\left(X_{i}, t_{i}\right)\right] \bar{X}_{2, i}\right. \\
& \left.+\left[K_{1}^{(j)}+K_{\mathrm{n}}^{(j)}\left(X_{i}, t_{i}\right)\right] \bar{X}_{1, i}+f_{\mathrm{e}}^{(j)}\left(t_{i}\right)\right\} \frac{h^{2}}{2}+\bar{R}_{1}^{(j)}
\end{aligned}
$$

where, $C_{1}^{(j)}, C_{\mathrm{n}}^{(j)}, K_{1}^{(j)}, K_{\mathrm{n}}^{(j)}$, respectively, denote the $j$ th row vectors of the matrices $C_{\mathrm{l}}, C_{\mathrm{n}}$, $K_{1}, K_{\mathrm{n}}$. For convenience in further discussion, let the transversally linearized velocity drift vector (as in Equation (5)) be denoted as $\bar{A}\left(\bar{X}_{1}, \bar{X}_{2}, t, X_{1, i}, X_{2, i}, t_{i}\right)=\left\{\bar{a}^{(j)} \mid j=1, \ldots, N\right\}$ so that the $j$ th scalar component is given by $\bar{a}^{(j)}=\left\{C_{1}^{(j)}+C_{\mathrm{n}}^{(j)}\right\} \bar{X}_{2}+\left\{K_{1}^{(j)}+K_{\mathrm{n}}^{(j)}\right\} \bar{X}_{1}+f_{\mathrm{e}}^{(j)}(t)$. Now, the displacement remainder corresponding to the LTL-based displacement map (21) is given by

$$
\begin{aligned}
\bar{R}_{1}^{(j)}= & \sum_{r=1}^{q} \int_{t_{i-1}}^{t_{i}} \int_{t_{i-1}}^{s} \int_{t_{i-1}}^{s_{1}} \dot{\sigma}_{r}^{(j)}\left(s_{2}\right) \mathrm{d} s_{2} \mathrm{~d} W_{r}\left(s_{1}\right) \mathrm{d} s \\
& +\sum_{r=1}^{q} \sum_{m=1}^{N} \int_{t_{i-1}}^{t_{i}} \int_{t_{i-1}}^{s} \int_{t_{i-1}}^{s_{1}} \sigma_{r}^{(m)}\left(s_{2}\right)\left\{C_{1}^{(j, m)}+C_{\mathrm{n}}^{(j, m)}\left(X_{i}, t_{i}\right)\right\} \mathrm{d} W_{r}\left(s_{2}\right) \mathrm{d} s_{1} \mathrm{~d} s
\end{aligned}
$$




$$
\begin{aligned}
& +\int_{t_{i-1}}^{t_{i}} \int_{t_{i-1}}^{s} \int_{t_{i-1}}^{s_{1}}\left[\dot{f}_{\mathrm{e}}^{(j)}\left(s_{2}\right)+\sum_{m=1}^{N} x_{2}^{(m)}\left(s_{2}\right)\left\{K_{1}^{(j, m)}+K_{\mathrm{n}}^{(j, m)}\left(X_{i}, t_{i}\right)\right\}\right. \\
& \left.+\sum_{m=1}^{N} \bar{a}^{(m)}\left\{C_{1}^{(j, m)}+C_{\mathrm{n}}^{(j, m)}\left(X_{i}, t_{i}\right)\right\}\right] \mathrm{d} s_{2} \mathrm{~d} s_{1} \mathrm{~d} s \\
& -\left[\sum_{r=1}^{q} \sum_{m=1}^{N} \int_{t_{i-1}}^{t_{i}} \sigma_{r}^{(m)}\left\{C_{1}^{(j, m)}+C_{\mathrm{n}}^{(j, m)}\left(X_{i}, t_{i}\right)\right\} \mathrm{d} W_{r}(s)\right] \frac{h^{2}}{2} \\
& -\left[\int _ { t _ { i - 1 } } ^ { t _ { i } } \left[\dot{f}_{\mathrm{e}}^{(j)}(s)+\sum_{m=1}^{N} x_{2}^{(m)}(s)\left\{K_{1}^{(j, m)}+K_{\mathrm{n}}^{(j, m)}\left(X_{i}, t_{i}\right)\right\}\right.\right. \\
& \left.\left.+\sum_{m=1}^{N} \bar{a}^{(m)}\left(X(s), s, X_{i}, t_{i}\right)\left\{C_{1}^{(j, m)}+C_{\mathrm{n}}^{(j, m)}\left(X_{i}, t_{i}\right)\right\}\right] \mathrm{d} s\right] \frac{h^{2}}{2}
\end{aligned}
$$

In the above expression, the scalar $C_{1}^{(j, m)}$ denotes the $(j, m)$ th element of the matrix $C_{1}$, and so on.

If the constraint conditions (6) along with the imposed identities in Equations 3(a-c) are now made use of in Equation (20), it is observed that the expansions for $x_{1, i}^{(j)}$ and $\bar{x}_{1, i}^{(j)}$, as obtained using Equations (18) and (20) respectively, are identical except for the remainder terms, viz $R_{1}^{(j)}$ and $\bar{R}_{1}^{(j)}$. Thus the error order for $\bar{x}_{1}^{(j)}$ is determined by the order of $E_{1, i}^{(j)}=R_{1}^{(j)}-\bar{R}_{1}^{(j)}$. Taking expectation of this term followed by the use of inequality (10) and Equation (15) leads to the following estimate (for some real constant vector $\bar{Q}=\left\{\bar{Q}^{(j)} \mid j=1, \ldots, N\right\}$ ):

$$
E\left(\left|E_{1, i}^{(j)}\right|\right) \leqslant \bar{Q}^{(j)}\left(1+\left\|X_{i-1}\right\|^{2}\right) h^{3}
$$

Thus, one has $r_{\mathrm{m}}=3.0$. Similarly, using inequality (11) and Equation (15) one has the following estimate of the mean square error:

$$
\left|E\left(E_{1, i}^{(l)}\right)^{2}\right|^{\frac{1}{2}} \leqslant \bar{Q}^{(l)}\left(1+\left\|X_{i-1}\right\|^{2}\right) h^{2.5}
$$

and thus $r_{\mathrm{s}}=2.5$. Now, a direct use of proposition (1) (noting that $r_{\mathrm{m}}=3.0=r_{\mathrm{s}}+\frac{1}{2}$ and $r_{\mathrm{s}}>\frac{1}{2}$ ) leads to the global error order $r_{\mathrm{g}}=2.0$ for the displacement components obtained via the LTL scheme proposed in this section. In a similar manner, one can expand the original and LTL-based velocity components, denoted respectively, as $x_{2, i}^{(j)}$ and $\bar{x}_{2, i}^{(j)}$, over the time step $h$ via the Ito-Taylor series to get

$$
x_{2, i}^{(j)}=x_{2, i-1}^{(j)}+\sum_{r=1}^{q} \sigma_{r}^{(j)}\left(t_{i-1}\right) I_{r}+a^{(j)}\left(X_{i}, t_{i}\right) h+R_{2}^{(j)}
$$


where

$$
\begin{aligned}
R_{2}^{(j)}= & \sum_{r=1}^{q} \int_{t_{i-1}}^{t_{i}} \int_{t_{i-1}}^{s} \dot{\sigma}_{r}^{(j)} \mathrm{d} s_{1} \mathrm{~d} W_{r}(s)+\sum_{r=1}^{q} \int_{t_{i-1}}^{t_{i}} \int_{t_{i-1}}^{s} \Lambda_{r} a^{(j)}\left(X\left(s_{1}\right), s_{1}\right) \mathrm{d} W_{r}\left(s_{1}\right) \mathrm{d} s \\
& +\int_{t_{i-1}}^{t_{i}} \int_{t_{i-1}}^{s} L a^{(j)}\left(X\left(s_{1}\right), s_{1}\right) \mathrm{d} s_{1} \mathrm{~d} s \\
& -\left[\sum_{r=1}^{q} \int_{t_{i-1}}^{t_{i}} \Lambda_{r} a^{(j)}(X(s), s) \mathrm{d} W_{r}(s)+\int_{t_{i-1}}^{t_{i}} L a^{(j)}(X(s), s) \mathrm{d} s\right] h
\end{aligned}
$$

and

$$
\begin{aligned}
\bar{x}_{2, i}^{(j)}= & x_{2, i-1}^{(j)}+\sum_{r=1}^{q} \sigma_{r}^{(j)}\left(t_{i-1}\right) I_{r}+\left\{\left[C_{1}^{(j)}+C_{\mathrm{n}}^{(j)}\left(X_{i}, t_{i}\right)\right] \bar{X}_{2, i}+\left[K_{1}^{(j)}+K_{\mathrm{n}}^{(j)}\left(X_{i}, t_{i}\right) \bar{X}_{1, i}\right]\right. \\
& \left.+f_{\mathrm{e}}^{(j)}\left(t_{i}\right)\right\} h+\bar{R}_{2}^{(j)}
\end{aligned}
$$

where,

$$
\begin{aligned}
\bar{R}_{2}^{(j)}= & \sum_{r=1}^{q} \int_{t_{i-1}}^{t_{i}} \int_{t_{i-1}}^{s} \dot{\sigma}_{r}^{(j)}\left(s_{1}\right) \mathrm{d} s_{1} \mathrm{~d} W_{r}(s)+\sum_{r=1}^{q} \sum_{m=1}^{N} \sigma_{r}^{(m)}\left\{C_{1}^{(j, m)}+C_{\mathrm{n}}^{(j, m)}\left(X_{1 i}, X_{2, i}, t_{i}\right)\right\} I_{r} 0 \\
& +\int_{t_{i-1}}^{t_{i-1}} \int_{t_{i-1}}^{s}\left[\dot{f}_{\mathrm{e}}^{(j)}\left(s_{1}\right)+\sum_{m=1}^{N} x_{2}^{(m)}\left(s_{1}\right)\left\{K_{1}^{(j, m)}+K_{\mathrm{n}}^{(j, m)}\left(X_{1 i}, X_{2 i}, t_{i}\right)\right\}\right. \\
& \left.+\sum_{m=1}^{N} \bar{a}^{(m)}\left(s_{1}\right)\left\{C_{1}^{(j, m)}+C_{\mathrm{n}}^{(j, m)}\left(X_{1 i}, X_{2 i}, t_{i}\right)\right\}\right] \mathrm{d} s_{1} \mathrm{~d} s \\
& -\left[\left\{\sum_{r=1}^{q} \sum_{m=1}^{N} \sigma_{r}^{(m)}\left\{C_{1}^{(j, m)}+C_{\mathrm{n}}^{(j, m)}\left(X_{1 i}, X_{2, i}, t_{i}\right)\right\} I_{r}\right\}\right. \\
& +\left\{\int _ { t _ { i - 1 } } ^ { t _ { i } } \left[\dot{f}_{\mathrm{e}}^{(j)}(s)+\sum_{m=1}^{N} x_{2}^{(m)}(s)\left\{K_{1}^{(j, m)}+K_{\mathrm{n}}^{(j, m)}\left(X_{1 i}, X_{2 i}, t_{i}\right)\right\}\right.\right. \\
& \left.\left.\left.+\sum_{m=1}^{N} \bar{a}^{(m)}(s)\left\{C_{1}^{(j, m)}+C_{\mathrm{n}}^{(j, m)}\left(X_{1 i}, X_{2 i}, t_{i}\right)\right\}\right] \mathrm{d} s\right\}\right] h
\end{aligned}
$$

As before, one observes that stochastic maps given by Equations (25) and (27) are identical except for the velocity remainders, $R_{2}^{(j)}$ and $\bar{R}_{2}^{(j)}$. Denoting $E_{2, i}^{(l)}=x_{2, i}^{(l)}-\bar{x}_{2, i}^{(l)}$, the following estimates are immediately derived:

$$
E\left(\left|E_{2, i}^{(l)}\right|\right) \leqslant \bar{Q}^{(l)}\left(1+\left\|X_{i-1}\right\|^{2}\right) h^{2}
$$




$$
\left|E\left(E_{2, i}^{(l)}\right)^{2}\right|^{1 / 2} \leqslant \bar{Q}^{(l)}\left(1+\left\|X_{i-1}\right\|^{2}\right) h^{3 / 2}
$$

Using proposition 1, it is clear that the local and global error orders for the LTL-based velocity vector, $\bar{X}_{2}$, are respectively, given by $r_{\mathrm{s}}=1.5$ and $r_{\mathrm{g}}=1.0$. Error orders for velocity components are therefore one order less than their corresponding values for the displacement components.

2.1.2. The case of multiplicative noises. Consider Equation (2) driven by sufficiently general $q$ multiplicative noise (diffusion) vectors $B_{r}\left(X_{1}, X_{2}, t\right)=\left\{b_{r}^{(j)}\left(X_{1}, X_{2}, t\right) \mid j=1, \ldots, n\right\}$, $r=1, \ldots, q$, which are functions of both displacement and velocity components. In other words, considering the decomposition, $B_{r}\left(X_{1}, X_{2}, t\right)=\sigma_{r}(t)+\mu_{r}\left(X_{1}, X_{2}, t\right)$, of the diffusion components, the noise will be multiplicative if at least one of the functions $\mu_{r}, r=1, \ldots, q$ is an explicit function of $X_{1}$ and/or $X_{2}$. Following the same approach as in Section 2.1.1, an implicit stochastic Taylor expansion for $x_{1, i}^{(j)}$ corresponding to Equation (2) may be written as

$$
x_{1, i}^{(j)}=x_{1, i-1}^{(j)}+x_{2, i-1}^{(j)} h+\sum_{r=1}^{q} b_{r}^{(j)}\left(X_{i}, t_{i}\right) I_{r 0}+a^{(j)}\left(X_{i}, t_{i}\right) h^{2} / 2+\Delta_{1}^{(j)}
$$

with the associated remainder

$$
\begin{aligned}
\Delta_{1}^{(j)}= & \sum_{p=1}^{q} \sum_{r=1}^{q} \int_{t_{i-1}}^{t_{i}} \int_{t_{i-1}}^{s} \int_{t_{i-1}}^{s_{1}} \Lambda_{p} b_{r}^{(j)}\left(X\left(s_{2}\right), s_{2}\right) \mathrm{d} W_{p}\left(s_{2}\right) \mathrm{d} W_{r}\left(s_{1}\right) \mathrm{d} s \\
& +\sum_{r=1}^{q} \int_{t_{i-1}}^{t_{i}} \int_{t_{i-1}}^{s} \int_{t_{i-1}}^{s_{1}} L b_{r}^{(j)}\left(X\left(s_{2}\right), s_{2}\right) \mathrm{d} s_{2} \mathrm{~d} W_{r}\left(s_{1}\right) \mathrm{d} s \\
& -\sum_{r=1}^{q}\left[\sum_{p=1}^{q} \int_{t_{i-1}}^{t_{i}} \Lambda_{p} b_{r}^{(j)}(X(s), s) \mathrm{d} W_{p}(s)+\int_{t_{i-1}}^{t_{i}} L a^{(j)}(X(s), s) \mathrm{d} s\right] I_{r} 0 \\
& +\sum_{r=1}^{q} \int_{t_{i-1}}^{t_{i}} \int_{t_{i-1}}^{s} \int_{t_{i-1}}^{s_{1}} \Lambda_{r} a^{(j)}\left(X\left(s_{2}\right), s_{2}\right) \mathrm{d} W_{r}\left(s_{2}\right) \mathrm{d} s_{1} \mathrm{~d} s+\int_{t_{i-1}}^{t_{i}} \int_{t_{i-1}}^{s} \int_{t_{i-1}}^{s_{1}} \\
& L a^{(j)}\left(X\left(s_{2}\right), s_{2}\right) \mathrm{d} s_{2} \mathrm{~d} s_{1} \mathrm{~d} s \\
& -\left[\sum_{r=1}^{q} \int_{t_{i-1}}^{t_{i}} \Lambda_{r} a^{(j)}(X(s), s) \mathrm{d} s+\int_{t_{i-1}}^{t_{i}} L a^{(j)}(X(s), s) \mathrm{d} s\right] \frac{h^{2}}{2}
\end{aligned}
$$

A corresponding expansion for the LTL-based displacement component, $\bar{x}_{1, i}^{(j)}$, leads to

$$
\begin{aligned}
\bar{x}_{1, i}^{(j)}= & x_{1, i-1}^{(j)}+x_{2, i-1}^{(j)} h+\sum_{r=1}^{q} b_{r}^{(j)}\left(X_{i}, t_{i}\right) I_{r 0}+\left[\left\{C_{1}^{(j)}+C_{\mathrm{n}}^{(j)}\left(X_{1 i}, X_{2 i}, t_{i}\right)\right\} \bar{X}_{2, i}\right. \\
& \left.+\left\{K_{1}^{(j)}+K_{\mathrm{n}}^{(j)}\left(X_{1 i}, X_{2 i}, t_{i}\right)\right\} \bar{X}_{1, i}\right] \frac{h^{2}}{2}+\bar{\Delta}_{1}^{(j)}
\end{aligned}
$$


The remainder is

$$
\begin{aligned}
\bar{\Delta}_{1}^{(j)}= & -\left[\sum_{r=1}^{q} \int_{t_{i-1}}^{t_{i}} \dot{b}_{r}^{(j)}\left(X_{1 i}, X_{2 i}, s\right) \mathrm{d} s\right] I_{r} 0 \\
& +\sum_{r=1}^{q} \int_{t_{i-1}}^{t_{i}} \int_{t_{i-1}}^{s} \int_{t_{i-1}}^{s_{1}} \dot{b}_{r}^{(j)}\left(X_{1 i}, X_{2 i}, s_{2}\right) \mathrm{d} s_{2} \mathrm{~d} W_{r}\left(s_{1}\right) \mathrm{d} s \\
& -\left[\sum_{r=1}^{q} \int_{t_{i-1}}^{t_{i}} \Lambda_{r} \bar{a}^{(j)} \mathrm{d} W_{r}(s)+\int_{t_{i-1}}^{t_{i}} L \bar{a}^{(j)} \mathrm{d} s\right] \frac{h^{2}}{2} \\
& +\sum_{r=1}^{q} \int_{t_{i-1}}^{t_{i}} \int_{t_{i-1}}^{s} \int_{t_{i-1}}^{s_{1}} \Lambda_{r} \bar{a}^{(j)} \mathrm{d} W_{r}\left(s_{2}\right) \mathrm{d} s_{1} \mathrm{~d} s \\
& +\int_{t_{i-1}}^{t_{i}} \int_{t_{i-1}}^{s} \int_{t_{i-1}}^{s_{1}} L \bar{a}^{(j)} \mathrm{d} s_{2} \mathrm{~d} s_{1} \mathrm{~d} s
\end{aligned}
$$

where the expressions for $\Lambda_{r} \bar{a}^{(j)}$ and $L \bar{a}^{(j)}$ may be written in the following expanded forms:

$$
\begin{aligned}
\Lambda_{r} \bar{a}^{(j)}\left(\bar{X}_{1}, \bar{X}_{2}, s, X_{1 i}, X_{2 i}\right)= & \sum_{m=1}^{N} b_{r}^{(m)}\left(X_{1 i}, X_{2 i}, s\right)\left\{C_{1}^{(j, m)}+C_{\mathrm{n}}^{(j, m)}\left(X_{1 i}, X_{2 i}, t_{i}\right)\right\} \\
L \bar{a}^{(j)}\left(\bar{X}_{1}, \bar{X}_{2}, s, X_{1 i}, X_{2 i}\right)= & \dot{f}_{\mathrm{e}}^{(j)}(s)+\sum_{m=1}^{N} \bar{x}_{2}^{(m)}(s)\left\{K_{1}^{(j, m)}+K_{\mathrm{n}}^{(j, m)}\left(X_{1 i}, X_{2 i}, t_{i}\right)\right\} \\
& +\sum_{m=1}^{N} \bar{a}^{(m)}\left(\bar{X}_{1}, \bar{X}_{2}, s, X_{1 i}, X_{2 i}\right)\left\{C_{1}^{(j, m)}+C_{\mathrm{n}}^{(j, m)}\left(X_{1 i}, X_{2 i}, t_{i}\right)\right\}
\end{aligned}
$$

With the displacement error vector defined as $E_{1, i}=\left\{E_{1, i}^{(j)}\right\}=\left\{x_{1, i}^{(j)}-\bar{x}_{1, i}^{(j)}\right\}, \quad j=1, \ldots, n$, one can readily see, using identities $(3 \mathrm{a}-\mathrm{c})$ and (6), that the stochastic Taylor expansions (31) and (33) are identical except for their respective remainders, $\Delta_{1}^{(j)}$ and $\bar{\Delta}_{1}^{(j)}$, so that one has $\left\{E_{1, i}^{(j)}\right\}=\left\{\Delta_{1}^{(j)}-\bar{\Delta}_{1}^{(j)}\right\}$. Based on propositions 1 and 2, one arrives at the following bounds:

$$
\begin{array}{r}
E\left(\left|E_{1, i}^{(j)}\right|\right) \leqslant \bar{Q}^{(j)}\left(1+\left\|X_{i-1}\right\|^{2}\right) h^{3} \\
\left|E\left(E_{1, i}^{(j)}\right)^{2}\right|^{\frac{1}{2}} \leqslant \bar{Q}^{(j)}\left(1+\left\|X_{i-1}\right\|^{2}\right) h^{2}
\end{array}
$$

and hence the local and global displacement errors are, respectively, $r_{\mathrm{s}}=2.0$ and $r_{\mathrm{g}}=1.5$, i.e. 0.5 less than their corresponding values in the purely additive case (see Section 2.1.1). Thus the accuracy of the present version of the LTL method is generally less under multiplicative noises. 
It is however interesting to note that in the specific case of multiplicative coefficients being functions of displacements alone, i.e. $b_{r}^{(j)}\left(X_{1}, X_{2}, t\right)=b_{r}^{(j)}\left(X_{1}, t\right)$, the first and third terms of order $O\left(h^{2}\right)$ in Equation (32) vanish (via Equation (13b)) and thus $r_{\mathrm{s}}=2.5$ and $r_{\mathrm{g}}=2.0$, which is the same as for the purely additive case. Expansions similar to (31)-(34) may also be derived in the same way for the velocity components, $x_{2, i}^{(j)}$ and $\bar{x}_{2, i}^{(j)}$, and their corresponding remainders, $\Delta_{2}^{(j)}$ and $\bar{\Delta}_{2}^{(j)}$. It may consequently be shown that the local and global error orders under a general set of multiplicative noise vectors are $r_{\mathrm{s}}=1.0$ and $r_{\mathrm{g}}=0.5$. However in the particular case of the multiplicative noise vectors depending only on $X_{1}$, these error orders are incremented by 0.5 to $r_{\mathrm{s}}=1.5$ and $r_{\mathrm{g}}=1.0$.

It is worth noting at this stage that, unlike the stochastic Heun method, no restrictions are presently imposed in this study on the number of independent noise processes driving the system for the above and following error estimates to hold true.

\section{HIGHER-ORDER STOCHASTIC LTL SYSTEMS}

The objective of constructing a higher-order LTL system is to increase the order of accuracy of a stochastic LTL-based response calculation. In other words, as compared with the lower-order LTL methods, more terms in the Ito-Taylor expansions of LTL-based displacement and velocity components should match with those for the original displacement and velocity components. In this study, this is achieved by appropriately augmenting the dimension of the given (non-linear) system (2) followed by the construction of LTL-based conditional vector fields corresponding to the augmented system. In what follows, a further elaboration on higher-order LTL systems is provided separately for additive and multiplicative noise inputs.

\subsection{The case of purely additive noises}

As in Section 2.1.1, let the additive diffusion coefficients be denoted as $B_{r}\left(X_{1}, X_{2}, t\right)=$ $\left\{\sigma_{r}^{(j)}(t) \mid j=1, \ldots, N\right\}$. Additionally, it is generally required that the drift elements $a^{(j)}\left(X_{1}, X_{2}, t\right)$ is at least $C^{1}$ in the velocity vector $X_{2}$ and $C^{2}$ in the displacement vector $X_{1}$. Now the augmented $3 N$ dimensional (augmented) dynamical system corresponding to the $2 N$ dimensional original system, as given by Equation (2), is written as

$$
\begin{aligned}
& \mathrm{d} x_{1}^{(j)}=x_{2}^{(j)} \mathrm{d} t \\
& \mathrm{~d} x_{2}^{(j)}=x_{3}^{(j)} \mathrm{d} t+\sum_{r=1}^{q} \sigma_{r}^{(j)} \mathrm{d} W_{r}(t) \\
& \mathrm{d} x_{3}^{(j)}=L a^{(j)}\left(X_{1}, X_{2}, t\right) \mathrm{d} t+\sum_{r=1}^{q} \Lambda_{r} a^{(j)}\left(X_{1}, X_{2}, t\right) \mathrm{d} W_{r}(t)
\end{aligned}
$$

where $x_{3}^{(j)}(t)=a^{(j)}\left(X_{1}, X_{2}, t\right)$ and $\Lambda_{r}$ and $L$ are, respectively, the drift and diffusion operators as described by Equations (13b) and (13c). Note that the augmented system, in addition to being higher dimensional, is driven by both additive and (artificially generated) multiplicative noise processes. The higher-order LTL (HLTL) system is now constructed by simply writing 
an LTL equation corresponding to Equation (37) as

$$
\begin{aligned}
\mathrm{d} \bar{x}_{1}^{(j)}= & \bar{x}_{2}^{(j)} \mathrm{d} t \\
\mathrm{~d} \bar{x}_{2}^{(j)}= & \bar{x}_{3}^{(j)} \mathrm{d} t+\sum_{r=1}^{q} \sigma_{r}^{(j)}(t) \mathrm{d} W_{r}(t) \\
\mathrm{d} \bar{x}_{3}^{(j)}= & \left\{\Gamma_{1}^{(j)}\left(X_{1 i}, X_{2 i}, t_{i}\right) \bar{X}_{1}+\Gamma_{2}^{(j)}\left(X_{1 i}, X_{2 i}, t_{i}\right) \bar{X}_{2}+\Gamma_{3}^{(j)}\left(X_{1 i}, X_{2 i}, t_{i}\right) \bar{X}_{3}\right\} \mathrm{d} t \\
& +\sum_{r=1}^{q} \Lambda_{r} a^{(j)}\left(X_{1 i}, X_{2 i}, t_{i}\right) \mathrm{d} W_{r}(t)
\end{aligned}
$$

where $\Gamma_{p}^{(j)}, p=1,2,3$ is the (N-dimensional) $j$ th row vector of the corresponding $N \times N$ matrices $\Gamma_{p}$. These matrices are so formed (conditionally) as to satisfy the vector identity

$$
L A\left(X_{1 i}, X_{2 i}, t_{i}\right)=\Gamma_{1}\left(X_{1 i}, X_{2 i}, t_{i}\right) X_{1 i}+\Gamma_{2}\left(X_{1 i}, X_{2 i}, t_{i}\right) X_{2 i}+\Gamma_{3}\left(X_{1 i}, X_{2 i}, t_{i}\right) X_{3 i}
$$

In the discussion to follow, the augmented state vectors for systems (37) and (38) will be referred to as $Y=\left\{X_{1}, X_{2}, X_{3}\right\}^{\mathrm{T}}$ and $\bar{Y}=\left\{\bar{X}_{1}, \bar{X}_{2}, \bar{X}_{3}\right\}^{\mathrm{T}}$, respectively. Since Equation (38) is conditionally linear with purely additive noises, its solution can be written via its fundamental solution matrix, $\hat{\Phi}$, as

$$
\bar{Y}(t)=\hat{\Phi}\left(Y_{i}, t_{i}, t\right)\left\{Y_{i-1}+\int_{t_{i-1}}^{t} \hat{\Phi}^{-1}\left(Y_{i}, t_{i}, s\right) \sum_{r=1}^{q} \hat{B}_{r}\left(X_{1 i}, X_{2 i}, t_{i}, s\right) \mathrm{d} W_{r}(s)\right\}
$$

where, $\hat{B}_{r}\left(X_{1 i}, X_{2 i}, t_{i}, t\right)=\left\{\{0\},\left\{\sigma_{r}^{(j)}(t)\right\},\left\{\Lambda_{r} a^{(j)}\left(X_{1 i}, X_{2 i}, t_{i}\right)\right\}\right\}^{\mathrm{T}}$ is the $3 N$ dimensional augmented, conditionally additive diffusion vector. The following expression is used to determine $\hat{\Phi}$ :

$$
\hat{\Phi}\left(Y_{i}, t_{i}, t\right)=\exp \left\{\left[\hat{M}\left(Y_{i}, t_{i}\right)\right]\left(t-t_{i-1}\right)\right\}
$$

The coefficient matrix $\hat{M}$ in the above expression is given by

$$
[\hat{M}]=\left[\begin{array}{ccc}
{[0]} & {[I]} & {[0]} \\
{[0]} & {[0]} & {[I]} \\
{\left[\Gamma_{1}\right]} & {\left[\Gamma_{2}\right]} & {\left[\Gamma_{3}\right]}
\end{array}\right]
$$

The conditionally linear solution (40) is subject to the following constraint identities at time $t=t_{i}$ :

$$
\bar{X}_{1 i}=X_{1 i}, \quad \bar{X}_{2 i}=X_{2 i} \quad \text { and } \quad \bar{X}_{3 i}=X_{3 i}
$$

and the following initial conditions:

$$
\bar{X}_{1, i-1}=X_{1, i-1}, \quad \bar{X}_{2, i-1}=X_{2, i-1}, \quad \bar{X}_{3, i-1}=A\left(X_{1, i-1}, X_{2, i-1}, t_{i-1}\right)
$$

If the constraint conditions (39) and (43) are satisfied, it may be readily verified that the vector fields of the non-linear and transversally linearized augmented dynamical systems, represented, respectively, by Equations (37) and (38), become (instantaneously) identical at $t=t_{i}$. Now, referring to the augmented system (37), it may be realized that the new equation for $X_{3}(t)$ 
essentially describes the tangent space dynamics of the drift vector, $A\left(X_{1}, X_{2}, t\right)$. Hence, the augmented LTL system (38) should apparently have a better sensitivity to the solution paths generated by the original system (2) when $B_{r}\left(X_{1}, X_{2}, t\right)=\left\{\sigma_{r}^{(j)}(t)\right\}$. That it is indeed so is also reflected in the error estimates that now follow.

3.1.1. Error estimates. The basic steps for obtaining the local and global error estimates remain essentially the same as in Section 2. To begin with, the $j$ th displacement component $x_{1 i}^{(j)}$ is implicitly Taylor expanded using Equation (37) (with $B_{r}\left(X_{1}, X_{2}, t\right)=\left\{\sigma_{r}^{(j)}(t)\right\}$ ) as

$$
\begin{aligned}
x_{1 i}^{(j)}= & x_{1, i-1}^{(j)}+x_{2, i-1}^{(j)} h+\sum_{r=1}^{q} \int_{t_{i-1}}^{t_{i}} \int_{t_{i-1}}^{s} \sigma_{r}^{(j)}\left(s_{1}\right) \mathrm{d} W_{r}\left(s_{1}\right) \mathrm{d} s+a^{(j)}\left(X_{1, i-1}, X_{2, i-1}, t_{i-1}\right) \frac{h^{2}}{2} \\
& +\sum_{r=1}^{q} \Lambda_{r} a^{(j)}\left(X_{1 i}, X_{2 i}, t_{i}\right) I_{r 00}+L a^{(j)}\left(X_{1 i}, X_{2 i}, t_{i}\right) \frac{h^{3}}{6}+\hat{R}_{1}^{(j)}
\end{aligned}
$$

with the remainder

$$
\begin{aligned}
\hat{R}_{1}^{(j)}= & \sum_{m=1}^{q} \sum_{r=1}^{q} \int_{t_{i-1}}^{t_{i}} \int_{t_{i-1}}^{s} \int_{t_{i-1}}^{s_{1}} \int_{t_{i-1}}^{s_{2}} \Lambda_{m} \Lambda_{r} a^{(j)}\left(X_{1}\left(s_{3}\right), X_{2}\left(s_{3}\right), s_{3}\right) \mathrm{d} W_{m}\left(s_{3}\right) \mathrm{d} W_{r}\left(s_{2}\right) \mathrm{d} s_{1} \mathrm{~d} s \\
& +\sum_{r=1}^{q} \int_{t_{i-1}}^{t_{i}} \int_{t_{i-1}}^{s} \int_{t_{i-1}}^{s_{1}} \int_{t_{i-1}}^{s_{2}} L \Lambda_{r} a^{(j)} \mathrm{d} s_{3} \mathrm{~d} W_{r}\left(s_{2}\right) \mathrm{d} s_{1} \mathrm{~d} s \\
& +\sum_{r=1}^{q} \int_{t_{i-1}}^{t_{i}} \int_{t_{i-1}}^{s} \int_{t_{i-1}}^{s_{1}} \int_{t_{i-1}}^{s_{2}} \Lambda_{r} L a^{(j)} \mathrm{d} W_{r}\left(s_{3}\right) \mathrm{d} s_{2} \mathrm{~d} s_{1} \mathrm{~d} s \\
& +\int_{t_{i-1}}^{t_{i}} \int_{t_{i-1}}^{s} \int_{t_{i-1}}^{s_{1}} \int_{t_{i-1}}^{s_{2}} L^{2} a^{(j)} \mathrm{d} s_{3} \mathrm{~d} s_{2} \mathrm{~d} s_{1} \mathrm{~d} s \\
& -\sum_{r=1}^{q}\left[\sum_{m=1}^{q}\left\{\int_{t_{i-1}}^{t_{i}} \Lambda_{r} \Lambda_{m} a^{(j)}\left(X_{1}(s), X_{2}(s), s\right) \mathrm{d} W_{m}(s)+\int_{t_{i-1}}^{t_{i}} \Lambda_{r} L a^{(j)} \mathrm{d} s\right\}\right] I_{r} 00 \\
& -\sum_{r=1}^{q}\left[\left\{\int_{t_{i-1}}^{t_{i}} L \Lambda_{r} a^{(j)}\left(X_{1}(s), X_{2}(s), s\right) \mathrm{d} W_{r}(s)+\int_{t_{i-1}}^{t_{i}} L^{2} a^{(j)} \mathrm{d} s\right\}\right] \frac{h^{3}}{6}
\end{aligned}
$$

Now expanding the transversally linearized $j$ th displacement component $\bar{x}_{1 i}^{(j)}$ using the higherorder LTL Equation (38) followed by the imposition of the constraint identities (39) and (43) and the initial conditions (44), finally leads to

$$
\begin{aligned}
\bar{x}_{1 i}^{(j)}= & x_{1, i-1}^{(j)}+x_{2, i-1}^{(j)} h+\sum_{r=1}^{q} \int_{t_{i-1}}^{t_{i}} \int_{t_{i-1}}^{s} \sigma_{r}^{(j)}\left(s_{1}\right) \mathrm{d} W_{r}\left(s_{1}\right) \mathrm{d} s+x_{3, i-1}^{(j)} \frac{h^{2}}{2} \\
& +\sum_{r=1}^{q} \Lambda_{r} a^{(j)}\left(X_{1 i}, X_{2 i}, t_{i}\right) I_{r 00}+L a^{(j)}\left(X_{1 i}, X_{2 i}, t_{i}\right) \frac{h^{3}}{6}+\tilde{R}_{1}^{(j)}
\end{aligned}
$$


Since $x_{3, i-1}^{(j)}=a^{(j)}\left(X_{i-1}, t_{i-1}\right)$, it is noted that the expansion for $\bar{x}_{1, i}^{(j)}$ matches with that for $x_{1 i}^{(j)}$ (Equation (45)) except for the remainder, $\tilde{R}_{1}^{(j)}$, which is presently given by

$$
\begin{aligned}
\tilde{R}_{1}^{(j)}= & \sum_{p=1}^{3} \sum_{r=1}^{q} \sum_{k=1}^{N}\left[\Gamma_{p}\right]_{j, k} \int_{t_{i-1}}^{t_{i}} \int_{t_{i-1}}^{s} \int_{t_{i-1}}^{s_{1}} \int_{t_{i-1}}^{s_{2}} \Lambda_{r} \bar{x}_{p}^{(k)}\left(s_{3}\right) \mathrm{d} W_{r}\left(s_{3}\right) \mathrm{d} s_{2} \mathrm{~d} s_{1} \mathrm{~d} s \\
& +\sum_{p=1}^{3} \sum_{k=1}^{N}\left[\Gamma_{p}\right]_{j, k} \int_{t_{i-1}}^{t_{i}} \int_{t_{i-1}}^{s} \int_{t_{i-1}}^{s_{1}} \int_{t_{i-1}}^{s_{2}} L \bar{x}_{p}^{(k)}\left(s_{3}\right) \mathrm{d} s_{3} \mathrm{~d} s_{2} \mathrm{~d} s_{1} \mathrm{~d} s \\
& -\sum_{p=1}^{3} \sum_{k=1}^{N}\left[\Gamma_{p}\right]_{l, k}\left\{\left(\sum_{r=1}^{q} \int_{t_{i-1}}^{t_{i}} \Lambda_{r} \bar{x}_{p}^{(j)} \mathrm{d} W_{r}(s)\right)+\int_{t_{i-1}}^{t_{i}} L \bar{x}_{p}^{(j)} \mathrm{d} s\right\} \frac{h^{3}}{6}
\end{aligned}
$$

Using propositions (1) and (2) on the signed error $E_{1, i}^{(j)}=\hat{R}_{1}^{(j)}-\tilde{R}_{1}^{(j)}$, it is now straightforward to show that $E\left(\left|E_{1, i}^{(j)}\right|\right) \equiv O\left(h^{4}\right)$ and $\left[E\left(\left|E_{1, i}^{(j)}\right|^{2}\right)\right]^{\frac{1}{2}} \equiv O\left(h^{3}\right)$. The local and global displacement error orders are therefore, respectively, given by $r_{\mathrm{s}}=3.0$ and $r_{\mathrm{g}}=2.5$. Carrying out a similar exercise for the velocity components, $x_{2, i}^{(j)}$ and $\bar{x}_{2, i}^{(j)}$, it is not difficult to show that the error orders in the HLTL-based velocity approximations are $r_{\mathrm{s}}=2.0$ and $r_{\mathrm{g}}=1.5$ It is also important to note that in the specific case, when the non-linearity in the drift vector is only due to displacements, i.e. $a^{(j)}\left(X_{1}, X_{2}, t\right)$ is linear in $X_{2}$ for all $j \in[1, N]$, then terms involving $\Lambda_{r} \Lambda_{m} a^{(j)}\left(X_{1}, X_{2}, t\right)$ become zero and thus one has an enhanced accuracy with $r_{\mathrm{s}}=3.5$ and $r_{\mathrm{g}}=3.0$ for displacements and $r_{\mathrm{s}}=2.5$ and $r_{\mathrm{g}}=2.0$ for velocities.

\subsection{The case of multiplicative noise inputs}

Construction of appropriate higher-order LTL equations under sufficiently general multiplicative excitations is not a simple task, especially when one strictly requires that the LTL-based drift and diffusions vectors be instantaneously identical with the original drift and diffusion vectors (say, at $t=t_{i}$ ). Such an identity is quite essential to show that, whenever the targeted solution is phase-independent (roughly meaning an asymptotic insensitivity of time histories to initial conditions), the LTL methods remain accurate even under arbitrarily high time step sizes [11]. If, on the other hand, the condition of the instantaneous identity of the original and LTL-based diffusion vectors is waived in favour of a 'higher accuracy' (purely in terms of the error orders expressed as exponents of $h$ ) in transient and phase-dependent regimes, then one can construct the following higher-order LTL equations for a given set of diffusion vectors $\left\{B_{r}\left(X_{1}, X_{2}, t\right)\right\}$ :

$$
\begin{aligned}
\mathrm{d} \bar{x}_{1}^{(j)}= & \bar{x}_{2}^{(j)} \mathrm{d} t \\
\mathrm{~d} \bar{x}_{2}^{(j)}= & \bar{x}_{3}^{(j)} \mathrm{d} t+\sum_{r=1}^{q} b_{r}^{(j)}\left(X_{1, i-1}, X_{2, i-1}, t_{i-1}\right) \mathrm{d} W_{r}(t) \\
& +\sum_{r=1}^{q} \sum_{u=1}^{q} \Lambda_{u} b_{r}^{(j)}\left(X_{1, i-1}, X_{2, i-1}, t_{i-1}\right)\left(W_{u}(t)-W_{u}\left(t_{i-1}\right)\right) \mathrm{d} W_{r}(t) \\
& +\sum_{r=1}^{q} L b_{r}^{(j)}\left(X_{1, i-1}, X_{2, i-1}, t_{i-1}\right) t \mathrm{~d} W_{r}(t)
\end{aligned}
$$




$$
\begin{aligned}
\mathrm{d} \bar{x}_{3}^{(j)}= & \left\{\Gamma_{1}^{(j)}\left(X_{1 i}, X_{2 i}, t_{i}\right) \bar{X}_{1}+\Gamma_{2}^{(j)}\left(X_{1 i}, X_{2 i}, t_{i}\right) \bar{X}_{2}+\Gamma_{3}^{(j)}\left(X_{1 i}, X_{2 i}, t_{i}\right) \bar{X}_{3}\right\} \mathrm{d} t \\
& +\sum_{r=1}^{q} \Lambda_{r} a^{(j)}\left(X_{1, i-1}, X_{2, i-1}, t_{i-1}\right) \mathrm{d} W_{r}(t)
\end{aligned}
$$

subject to initial conditions $\bar{x}_{1, i-1}^{(j)}=x_{1, i-1}^{(j)}, \bar{x}_{2, i-1}^{(l)}=x_{2, i-1}^{(l)}$ and $\bar{x}_{3, i-1}^{(j)}=a^{(j)}\left(X_{1, i-1}, X_{2, i-1}\right.$, $\left.t_{i-1}\right)$ as well as the same constraint identities as in Equations (39) and (43). With the fundamental solution matrix, $\hat{\Phi}$, defined as in Equation (41) and the coefficient matrix, $[\hat{M}]$, as in Equation (42), the conditionally linear solution, $\bar{Y}=\left\{\bar{X}_{1}, \bar{X}_{2}, \bar{X}_{3}\right\}^{\mathrm{T}}$, may presently be written as

$$
\bar{Y}(t)=\hat{\Phi}\left(Y_{i}, t_{i}, t\right)\left\{Y_{i-1}+\int_{t_{i-1}}^{t} \hat{\Phi}^{-1}\left(Y_{i}, t_{i}, s\right) \sum_{r=1}^{q} \tilde{B}_{r} \mathrm{~d} W_{r}(s)\right\}
$$

where the $3 N$ dimensional augmented diffusion vector $\tilde{B}_{r}\left(X_{1, i-1}, X_{2, i-1}, t_{i-1}\right)$ is given by

$$
\left\{\tilde{B}_{r}\right\}=\left\{\begin{array}{c}
\{0\} \\
\left\{B_{r, i-1}^{(j)}+\sum_{u=1}^{q} \Lambda_{u} B_{r, i-1}^{(j)}\left(W_{u}(t)-W_{u}\left(t_{i-1}\right)\right)+L B_{r, i-1}^{(j)} t\right\} \\
\left\{\Lambda_{r} a^{(j)}\left(X_{1, i-1}, X_{2, i-1}, t_{i-1}\right)\right\}
\end{array}\right\}
$$

In the above expression, $\{0\}$ is an $N$-dimensional zero vector and $b_{r, i-1}^{(j)}=b_{r}^{(j)}\left(X_{1, i-1}, X_{2, i-1}\right.$, $\left.t_{i-1}\right)$. Now, use of the transversal intersection condition (43) on the LHS of Equation (50) (at $t=t_{i}$ ) leads to $3 N$ non-linear algebraic in the $3 N$ unknowns $\left\{X_{1 i}, X_{2 i}, X_{3 i}\right\}^{\mathrm{T}}$. Further computational details for performing the integrations with respect to the Wiener increments will be briefly outlined in the next section. It may be noted that the augmented diffusion vector $\tilde{B}_{r}$ in the LTL Equation (49) is not a function of $\left(X_{1 i}, X_{2 i}, t_{i}\right)$ and hence the conditional vector field in Equation (49) cannot instantaneously match with the original vector field at $t=t_{i}$. In other words, the condition (43) for transversal intersections of the linearized and non-linear manifolds in the augmented phase space ( $3 n$ dimensional) cannot be perfectly satisfied.

3.2.1. Error estimates. As in the earlier cases, an implicit Ito-Taylor expansion of the displacement component, $x_{1 i}^{(j)}$, in terms of the original drift and diffusion fields, as in Equation (2), is given by

$$
\begin{aligned}
x_{1 i}^{(j)}= & x_{1, i-1}^{(j)}+x_{2, i-1}^{(j)} h+\sum_{r=1}^{q} B_{r, i-1}^{(j)} I_{r 0}+\sum_{r=1}^{q} \sum_{u=1}^{q} \Lambda_{u} B_{r, i-1}^{(j)} I_{u r 0}+\sum_{r=1}^{q} L B_{r, i-1}^{(j)} I_{0 r 0} \\
& +a^{(j)}\left(X_{1, i-1}, X_{2, i-1}, t_{i-1}\right) \frac{h^{2}}{2}+\sum_{r=1}^{q} \Lambda_{r} a^{(j)}\left(X_{1, i-1}, X_{2, i-1}, t_{i-1}\right) I_{r 00} \\
& +L a^{(j)}\left(X_{1 i}, X_{2 i}, t_{i}\right) \frac{h^{3}}{6}+\breve{R}_{1}^{(j)}
\end{aligned}
$$


Note that $L a^{(j)}\left(X_{1 i}, X_{2 i}, t_{i}\right)$ is the only implicit term in the above expansion. Presently, the remainder $\breve{R}_{1}^{(j)}$ is given by

$$
\begin{aligned}
\breve{R}_{1}^{(j)}= & \sum_{r=1}^{q} \sum_{u=1}^{q} \sum_{k=1}^{q} \int_{t_{i-1}}^{t_{i}} \int_{t_{i-1}}^{s} \int_{t_{i-1}}^{s_{1}} \int_{t_{i-1}}^{s_{2}} \Lambda_{k} \Lambda_{u} B_{r, s_{3}}^{(j)} \mathrm{d} W_{k}\left(s_{3}\right) \mathrm{d} W_{u}\left(s_{2}\right) \mathrm{d} W_{r}\left(s_{1}\right) \mathrm{d} s \\
& +\sum_{r=1}^{q} \sum_{u=1}^{q} \int_{t_{i-1}}^{t_{i}} \int_{t_{i-1}}^{s} \int_{t_{i-1}}^{s_{1}} \int_{t_{i-1}}^{s_{2}} L \Lambda_{u} B_{r, s_{3}}^{(j)} \mathrm{d} s_{3} \mathrm{~d} W_{u}\left(s_{2}\right) \mathrm{d} W_{r}\left(s_{1}\right) \mathrm{d} s \\
& +\sum_{r=1}^{q} \sum_{u=1}^{q} \int_{t_{i-1}}^{t_{i}} \int_{t_{i-1}}^{s} \int_{t_{i-1}}^{s_{1}} \int_{t_{i-1}}^{s_{2}} \Lambda_{u} L B_{r, s_{3}}^{(j)} \mathrm{d} W_{u}\left(s_{3}\right) \mathrm{d} s_{2} \mathrm{~d} W_{2}\left(s_{1}\right) \mathrm{d} s \\
& +\sum_{r=1}^{q} \int_{t_{i-1}}^{t_{i}} \int_{t_{i-1}}^{s} \int_{t_{i-1}}^{s_{1}} \int_{t_{i-1}}^{s_{2}} L^{2} B_{r, s_{3}}^{(j)} \mathrm{d} s_{3} \mathrm{~d} s_{2} \mathrm{~d} W_{2}\left(s_{1}\right) \mathrm{d} s \\
& +\sum_{r=1}^{q} \sum_{u=1}^{q} \int_{t_{i-1}}^{t_{i}} \int_{t_{i-1}}^{s} \int_{t_{i-1}}^{s_{1}} \int_{t_{i-1}}^{s_{2}} \Lambda_{u} \Lambda_{r} a_{s_{3}}^{(j)} \mathrm{d} W_{u}\left(s_{3}\right) \mathrm{d} W_{r}\left(s_{2}\right) \mathrm{d} s_{1} \mathrm{~d} s \\
& +\sum_{r=1}^{q} \int_{t_{i-1}}^{t_{i}} \int_{t_{i-1}}^{s} \int_{t_{i-1}}^{s_{1}} \int_{t_{i-1}}^{s_{2}} L \Lambda_{r} a_{s_{3}}^{(j)} \mathrm{d} s_{3} \mathrm{~d} W_{r}\left(s_{2}\right) \mathrm{d} s_{1} \mathrm{~d} s \\
& +\sum_{r=1}^{q} \int_{t_{i-1}}^{t_{i}} \int^{s} \int_{t_{i-1}}^{s_{1}} \int_{t_{i-1}}^{s_{2}} \Lambda_{r} L a_{s_{3}}^{(j)} \mathrm{d} W\left(s_{3}\right) \mathrm{d} s_{2} \mathrm{~d} s_{1} \mathrm{~d} s \\
& +\int_{t_{i-1}}^{t_{i}} \int_{t_{i-1}}^{s} \int_{t_{i-1}}^{s_{1}} \int_{t_{i-1}}^{s_{2}} L^{2} a_{s_{3}}^{(j)} \mathrm{d} s_{3} \mathrm{~d} s_{2} \mathrm{~d} s_{1} \mathrm{~d} s \\
& -\left[\sum_{r=1}^{q} \int_{t_{i-1}}^{t_{i}} \Lambda_{r} a_{s}^{(j)} \mathrm{d} W_{r}(s)+\int_{t_{i-1}}^{t_{i}} L a_{s}^{(j)} \mathrm{d} s\right] \frac{h^{3}}{6}
\end{aligned}
$$

The following abbreviated notations have been used in the above expression: $B_{r, s_{3}}^{(j)}=B_{r}^{(j)}\left(X_{1}\right.$ $\left.\left(s_{3}\right), X_{2}\left(s_{3}\right), s_{3}\right), a_{s_{3}}^{(j)}=a^{(j)}\left(X_{1}\left(s_{3}\right), X_{2}\left(s_{3}\right), s_{3}\right)$ and $a_{s}^{(j)}=a^{(j)}\left(X_{1}(s), X_{2}(s), s\right)$. A similar Ito-Taylor expansion of the linearized displacement component, $\bar{x}_{1 i}^{(j)}$, via the conditionally linear drift and diffusion fields as in Equation (49), followed by imposition of the appropriate constraint conditions may be seen to lead to the same series as in Equation (52) except for the remainder term. This conditional remainder, denoted by $\widehat{R}_{1}^{(j)}$, is given by

$$
\begin{aligned}
\widehat{R}_{1}^{(j)}= & \sum_{p=1}^{3} \sum_{k=1}^{N}\left[\Gamma_{p}\right]_{l k}\left\{\sum_{r=1}^{q} \int_{t_{i-1}}^{t_{i}} \int_{t_{i-1}}^{s} \int_{t_{i-1}}^{s_{1}} \int_{t_{i-1}}^{s_{2}} \Lambda_{r} \bar{x}_{p}^{(k)} \mathrm{d} W_{r}\left(s_{3}\right) \mathrm{d} s_{2} \mathrm{~d} s_{1} \mathrm{~d} s\right. \\
& \left.+\int_{t_{i-1}}^{t_{i}} \int_{t_{i-1}}^{s} \int_{t_{i-1}}^{s_{1}} \int_{t_{i-1}}^{s_{2}} L \bar{x}_{p}^{(k)} \mathrm{d} s_{3} \mathrm{~d} s_{2} \mathrm{~d} s_{1} \mathrm{~d} s\right\} \\
& -\left\{\sum_{p=1}^{3} \sum_{k=1}^{N}\left[\Gamma_{p}\right]_{l k}\left(\sum_{r=1}^{q} \int_{t_{i-1}}^{t_{i}} \Lambda_{r} \bar{x}_{p}^{(k)} \mathrm{d} W_{r}(s)+\int_{t_{i-1}}^{t_{i}} L \bar{x}_{p}^{(k)} \mathrm{d} s\right)\right\} \frac{h^{3}}{6}
\end{aligned}
$$


It may now be readily seen that the local and global error orders for the displacement vector via this version of HLTL are $r_{\mathrm{s}}=2.5$ and $r_{\mathrm{g}}=2.0$, respectively. Similar calculations for the velocity vector indicate the local and global error orders, respectively to be $r_{\mathrm{s}}=1.5$ and $r_{\mathrm{g}}=1.0$. However it must be noted that these estimates should often turn out to be conservative in view of the fact that in many non-linear engineering systems, the diffusion coefficients $B_{r}^{(j)}\left(X_{1}, X_{2}, t\right)$ are either independent of or linearly dependent on the velocity vector $X_{2}$. In such cases, terms involving the composition $\Lambda_{u} \circ \Lambda_{r}$ of the diffusion operators in the remainder equation (53) vanish. The local and global error orders thus become $r_{\mathrm{s}}=3.0$ and $r_{\mathrm{g}}=2.5$ for the computed displacement vector and $r_{\mathrm{s}}=2.0$ and $r_{\mathrm{g}}=1.5$ for the computed velocity vector.

\section{CERTAIN COMPUTATIONAL ISSUES}

Computations of the fundamental solution matrix and its inverse, crucial for the construction of a linearized solution, require exponentiation of certain (possibly quite large) system matrices. Let it be required to numerically obtain $\left[\Phi\left(t, t_{i-1}\right)=\exp \left\{[M]\left(t-t_{i-1}\right)\right\}\right.$. A computationally expedient way to do so is to divide the interval $\left(t_{i-1}, t\right]$ into $2^{k}$ equal sub-intervals and make use of the following identity:

$$
\Phi\left(t, t_{i-1}\right)=\left\{\Phi\left(\left(t_{i-1}+\frac{t-t_{i-1}}{2^{k}}\right), t_{i-1}\right)\right\}^{k}
$$

Denoting the RHS of the above equation as $\left\{\Phi_{k}\left(t_{i-1}\right)\right\}^{k}$ and $h_{k}=2^{-k}\left(t-t_{i-1}\right)$, one may use a deterministic Taylor expansion followed by the retention of the first few terms (may be four or even fewer) to compute $\Phi_{k}\left(t_{i-1}\right)$ as

$$
\Phi_{k}\left(t_{i-1}\right)=I+[M] h_{k}+[M]^{2} h_{k}^{2} / 2+\cdots
$$

where $[I]$ is an $n \times n$ identity matrix. A similar scheme may also be utilized to obtain the inverse $\Phi^{-1}\left(t, t_{i-1}\right)=\exp \left\{-[M]\left(t-t_{i-1}\right)\right\}$. In this case,

$$
\Phi_{k}^{-1}\left(t_{i-1}\right)=I-[M] h_{k}+[M]^{2} h_{k}^{2} / 2+\cdots
$$

At the time that this article was being written up, the attention of the author was attracted to a recent paper by Leung [12], where a similar matrix exponentiation as outlined in this section has been utilized for the response calculation of linear engineering systems under deterministic and random loading conditions.

Implementation of the linearization methods requires an appropriate modelling of the vector Wiener process $\left\{W_{r}(t) \mid r=1, \ldots, q\right\}$. This is numerically done by generating a set of pseudorandom vectors $\left\{g_{i}^{(r)} \mid r=1, \ldots, q\right\}$ for every positive integer $i$ (including zero) corresponding to the time interval $t_{i}$. Each element $g_{i}^{(r)}$ of the $i$ th vector is an independently generated $N(0,1)$ random number. One thus has

$$
\Delta W_{r, i}=W_{r}\left(t_{i}\right)-W_{r}\left(t_{i-1}\right)=g_{i}^{(r)} \sqrt{h}
$$

where $h=t_{i}-t_{i-1}$ is the uniform time step size. 
One may also note from Equations (7), (40) and (50) that the construction of particular solutions for the linearized equations involves determining the Gaussian stochastic integrals of the forms $\int_{t_{i-1}}^{t_{i}} \Psi(s) \mathrm{d} W_{r}(s)$ and $\int_{t_{i-1}}^{t_{i}} \Psi(s) W_{u}(s) \mathrm{d} W_{r}(s)$ for $u, r=1,2, \ldots, q$. Interpreted according to Ito, expectations of both these Gaussian integrals are zero. Moreover, one has for the standard deviation of the first integral

$$
\left.\sigma_{1}=\left\{E\left(\left|\int_{t_{i-1}}^{t_{i}} \Psi(s) \mathrm{d} W_{r}(s)\right|^{2}\right)\right\}^{1 / 2}=\left\{\int_{t_{i-1}}^{t_{i}} \Psi^{2}(s) \mathrm{d} s\right\}\right\}^{1 / 2}
$$

In this work, the RHS of the above equation is generated using a 3-point Gauss quadrature and finally the stochastic integral is obtained as $\int_{t_{i-1}}^{t_{i}} \Psi(s) \mathrm{d} W_{r}(s)=g_{i}^{(r)} \sigma_{1}$. In a similar manner, following Ito's definition, the standard deviation for the second integral is given by

$$
\left.\sigma_{2}=\left\{E\left(\left|\int_{t_{i-1}}^{t_{i}} \Psi(s) W_{u}(s) \mathrm{d} W_{r}(s)\right|^{2}\right)\right\}^{1 / 2}=\left\{\int_{t_{i-1}}^{t_{i}} \Psi^{2}(s) s \mathrm{~d} s\right\}\right\}^{1 / 2}
$$

Hence $\sigma_{2}$ may also be determined using a Gauss quadrature rule and the second integral is obtained as $\int_{t_{i-1}}^{t_{i}} \Psi(s) W_{u}(s) \mathrm{d} W_{r}(s)=g_{i}^{(u)} g_{i}^{(r)} \sigma_{2}$.

\section{NUMERICAL ILLUSTRATIONS}

Even though the focus of the present work is on the propositions and theoretical error estimates of a family of transversal linearization schemes, a rather limited numerical illustration on the stochastic response of a couple of oscillators is provided here. The construction of the corresponding LTL equations and their respective solutions may be done based on Sections 2 and 3 and such details are presently omitted for brevity. The first example problem concerns a single-degree-of-freedom (SDOF) hardening Duffing equation driven by combined additive and multiplicative noises, in addition to a deterministic periodic excitation. The equation of motion is presently written in an incremental form as

$$
\begin{aligned}
& \mathrm{d} x_{1}(t)=x_{2}(t) \mathrm{d} t \\
& \mathrm{~d} x_{2}(t)=\left\{-2 \pi \varepsilon_{1} x_{2}-4 \pi^{2} \varepsilon_{2}\left(x_{1}^{2}+1\right) x_{1}+4 \pi^{2} \varepsilon_{3} \cos (2 \pi t)\right\} \mathrm{d} t+g_{1} \mathrm{~d} W_{1}(t)-g_{2} x_{1} \mathrm{~d} W_{2}(t)
\end{aligned}
$$

In order to illustrate the construction of the transversally linearized vector fields corresponding to the above equation, the case of $g_{2}=0$ is first considered. This corresponds to the oscillator being driven only by a combination of a sinusoidal (deterministic) excitation and an additive (white noise) excitation of constant intensity $g_{1}$. For this case, the basic LTL-based linearized form, valid over the interval $\left(t_{i-1}, t_{i}\right]$, is

$$
\begin{aligned}
& \mathrm{d} \bar{x}_{1}(t)=\bar{x}_{2}(t) \mathrm{d} t \\
& \mathrm{~d} \bar{x}_{2}(t)=\left\{-2 \pi \varepsilon_{1} \bar{x}_{2}-4 \pi^{2} \varepsilon_{2}\left(x_{1 i}^{2}+1\right) \bar{x}_{1}+4 \pi^{2} \varepsilon_{3} \cos (2 \pi t)\right\} \mathrm{d} t+g_{1} \mathrm{~d} W_{1}(t)
\end{aligned}
$$

Referring to Equations (2), (3) and (5) in Section 2, it may be readily verified that $C_{1}=-2 \pi \varepsilon_{1}$, $C_{\mathrm{n}}=0, K_{1}=-4 \pi^{2} \varepsilon_{2}, K_{\mathrm{n}}=-4 \pi^{2} \varepsilon_{2} x_{1}^{2}$ for the present problem. Treating $x_{1 i}^{2}=\left(x\left(t_{i}\right)\right)^{2}$ as an 
D. ROY

unknown constant, the system coefficient matrix corresponding to the transversally linearized SDE (62) may be written as

$$
[M]=\left[\begin{array}{cc}
0 & 1 \\
-4 \pi^{2} \varepsilon_{2}\left(x_{1 i}^{2}+1\right) & -2 \pi \varepsilon_{1}
\end{array}\right]
$$

Following Equation (8), the fundamental solution matrix (FSM) for Equation (62), valid over $\left(t_{i-1}, t_{i}\right]$, may be conditionally constructed as

$$
\Phi\left(x_{1 i}, t, t_{i-1}\right)=\exp \left\{[M]\left(t-t_{i-1}\right)\right\}
$$

It may be noted that, for the presently chosen oscillator, the FSM is a function of $x_{1 i}$ alone (and not of both $x_{1 i}$ and $x_{2 i}$, as in the more general case). Using Equation (7), the displacement solution of Equation (62) may be written as

$$
\begin{aligned}
\bar{x}_{1 i}= & \Phi_{11, i} x_{1, i-1}+\Phi_{12, i} x_{2, i-1}+4 \pi^{2} \varepsilon_{3}\left[\Phi_{11, i} \int_{t_{i-1}}^{t_{i}} \Phi_{12, s}^{-1} \cos (2 \pi s) \mathrm{d} s\right. \\
& \left.+\Phi_{12, i} \int_{t_{i-1}}^{t_{i}} \Phi_{22, s}^{-1} \cos (2 \pi s) \mathrm{d} s\right] \\
& +g_{1}\left[\Phi_{11, i} \int_{t_{i-1}}^{t_{i}} \Phi_{12, s}^{-1} \mathrm{~d} W_{1}(s)+\Phi_{12, i} \int_{t_{i-1}}^{t_{i}} \Phi_{22, s}^{-1} \mathrm{~d} W_{1}(s)\right]
\end{aligned}
$$

where $\Phi_{p k, i}=\Phi_{p k}\left(x_{1 i}, t_{i}, t_{i-1}\right)$ and $\Phi_{p k, s}^{-1}=\Phi_{p k}^{-1}\left(x_{1 i}, s, t_{i-1}\right) ; p, k=1,2$. Now, if the transversal intersection condition (for displacements alone), i.e. $x_{1 i}=\bar{x}_{1 i}$, is made use of on the LHS of Equation (65), one gets a non-linear algebraic equation to solve for $x_{1 i}$. Once $x_{1 i}$ is computed, the velocity variable $x_{2 i}$ may be readily obtained from the following explicit map:

$$
\begin{aligned}
x_{2 i}= & \Phi_{21, i} x_{1, i-1}+\Phi_{22, i} x_{2, i-1}+4 \pi^{2} \varepsilon_{3}\left[\Phi_{21, i} \int_{t_{i-1}}^{t_{i}} \Phi_{12, s}^{-1} \cos (2 \pi s) \mathrm{d} s\right. \\
& \left.+\Phi_{22, i} \int_{t_{i-1}}^{t_{i}} \Phi_{22, s}^{-1} \cos (2 \pi s) \mathrm{d} s\right] \\
& +g_{1}\left[\Phi_{21, i} \int_{t_{i-1}}^{t_{i}} \Phi_{12, s}^{-1} \mathrm{~d} W_{1}(s)+\Phi_{22, i} \int_{t_{i-1}}^{t_{i}} \Phi_{22, s}^{-1} \mathrm{~d} W_{1}(s)\right]
\end{aligned}
$$

If it is intended to use the higher-order LTL method to transversally linearize the Duffing oscillator under additive, random excitation (i.e. $g_{2}=0$ ), then, following Equations (37) and 
(38), the higher-order LTL-based linearized SDEs over $\left(t_{i-1}, t_{i}\right]$ take the form

$$
\begin{aligned}
\mathrm{d} \bar{x}_{1}= & \bar{x}_{2} \mathrm{~d} t \\
\mathrm{~d} \bar{x}_{2}= & \bar{x}_{3} \mathrm{~d} t+g_{1} \mathrm{~d} W_{1}(t) \\
\mathrm{d} \bar{x}_{3}= & {\left[4 \pi^{2}\left\{\varepsilon_{1}^{2}-\varepsilon_{3}\left(1+3 x_{1 i}^{2}\right)\right\} \bar{x}_{2}+8 \pi^{2} \varepsilon_{1} \varepsilon_{3}\left(1+x_{1 i}^{2}\right) \bar{x}_{1}-8 \pi^{3} \varepsilon_{3}\left(\varepsilon_{1} \cos 2 \pi t+\varepsilon_{3} \sin 2 \pi t\right)\right] \mathrm{d} t } \\
& -2 \pi \varepsilon_{1} g_{1} \mathrm{~d} W_{1}(t)
\end{aligned}
$$

The system coefficient matrix is presently given by

$$
[M]=\left[\begin{array}{ccc}
0 & 1 & 0 \\
0 & 0 & 1 \\
8 \pi^{2} \varepsilon_{1} \varepsilon_{3}\left(1+x_{1 i}^{2}\right) & 4 \pi^{2}\left\{\varepsilon_{1}^{2}-\varepsilon_{3}\left(1+3 x_{1 i}^{2}\right)\right\} & 0
\end{array}\right]
$$

and the fundamental solution matrix is given by Equation (64). The rest of the procedure, i.e. that of constructing the conditional solution of the linearzed SDEs (67) and forming the non-linear algebraic equation for $x_{1 i}$, remains the same as in the lower-order case.

Now consider the more general case of the oscillator driven by combined additive and multiplicative, random excitations (i.e. $g_{1} \neq 0, g_{2} \neq 0$ ). Following the discussion in Section 2 , the lower-order version of the transversally linearized oscillator, valid over $\left(t_{i-1}, t_{i}\right]$, is given by

$$
\begin{aligned}
& \mathrm{d} \bar{x}_{1}(t)=\bar{x}_{2}(t) \mathrm{d} t \\
& \mathrm{~d} \bar{x}_{2}(t)=\left\{-2 \pi \varepsilon_{1} \bar{x}_{2}-4 \pi^{2} \varepsilon_{2}\left(x_{1 i}^{2}+1\right) \bar{x}_{1}+4 \pi^{2} \varepsilon_{3} \cos (2 \pi t)\right\} \mathrm{d} t+g_{1} \mathrm{~d} W_{1}(t)-g_{2} x_{1 i} \mathrm{~d} W_{2}(t)
\end{aligned}
$$

It may be noted that the multiplicative excitation is converted to a conditionally additive excitation in the lower-order LTL. The remaining set of procedures to determine the solution is precisely the same as followed for the additive case via the lower-order LTL method. On the other hand, the higher-order LTL-based linearized SDEs may be derived using Equation (49) as

$$
\begin{aligned}
\mathrm{d} \bar{x}_{1}= & \bar{x}_{2} \mathrm{~d} t \\
\mathrm{~d} \bar{x}_{2}= & \bar{x}_{3} \mathrm{~d} t+g_{1} \mathrm{~d} W_{1}(t)-g_{2}\left(x_{1, i-1}+x_{2, i-1}\right) \mathrm{d} W_{2}(t) \\
\mathrm{d} \bar{x}_{3}= & {\left[4 \pi^{2}\left\{\varepsilon_{1}^{2}-\varepsilon_{3}\left(1+3 x_{1 i}^{2}\right)\right\} \bar{x}_{2}+8 \pi^{2} \varepsilon_{1} \varepsilon_{3}\left(1+x_{1 i}^{2}\right) \bar{x}_{1}-8 \pi^{3} \varepsilon_{3}\left(\varepsilon_{1} \cos 2 \pi t+\varepsilon_{3} \sin 2 \pi t\right)\right] \mathrm{d} t } \\
& -2 \pi \varepsilon_{1}\left\{g_{1} \mathrm{~d} W_{1}(t)-g_{2} x_{1, i-1} \mathrm{~d} W_{2}(t)\right\}
\end{aligned}
$$

Once more, one arrives at a three-dimensional linearized SDE driven by conditionally additive white noise excitations. However, unlike the lower-order linearization given by Equation (69), the (conditionally) additive noise co-efficients in Equation (70) are unconditionally known. The system coefficient matrix in Equation (70) is given by Equation (68) and the rest of the solution procedure is also straightforward.

First, under a purely additive noise, comparisons of displacement and velocity histories via the basic LTL (BLTL) and the higher-order LTL (HLTL) are shown in Figure 1. In Figure 2, 

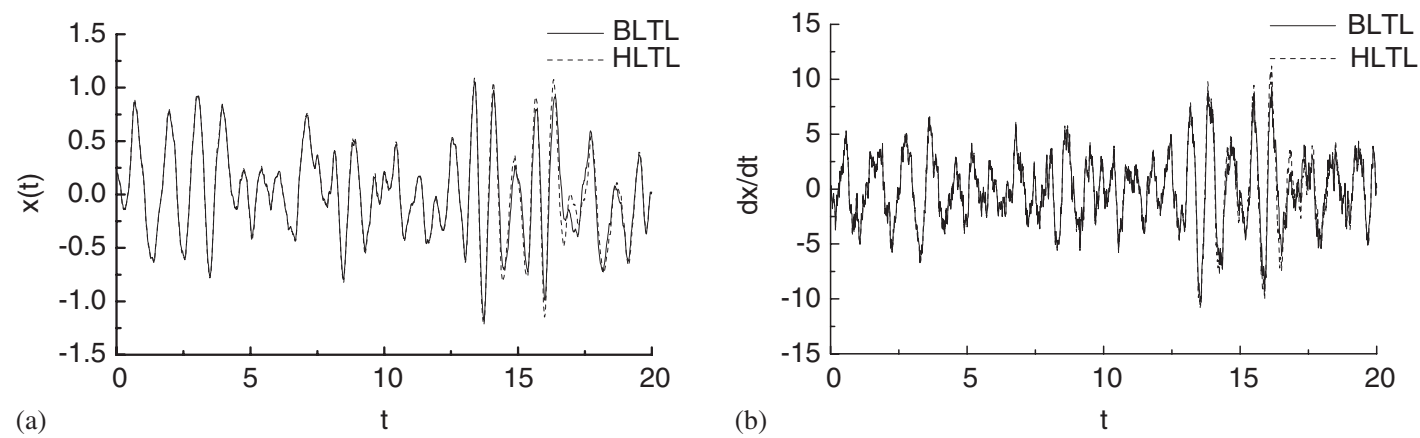

Figure 1. (a) Displacement history of the Duffing equation under only additive noise; $\varepsilon_{1}=0.25, \quad \varepsilon_{2}=1.0, \quad \varepsilon_{3}=0.0, \quad g_{1}=6.0, \quad g_{2}=0.0 ;$ and (b) velocity history of the Duffing equation under only additive noise; $\varepsilon_{1}=0.25, \varepsilon_{2}=1.0$, $\varepsilon_{3}=0.0, g_{1}=6.0, \quad g_{2}=0.0$.
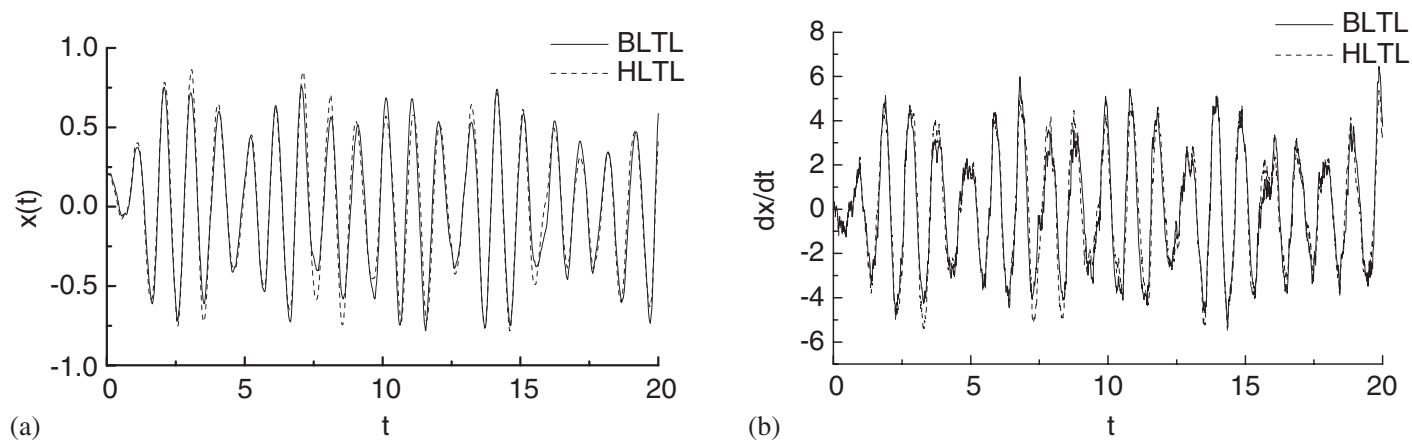

Figure 2. (a) Displacement history of the Duffing equation under additive and multiplicative noises along with a periodic excitation; $\varepsilon_{1}=0.25, \quad \varepsilon_{2}=1.0, \quad \varepsilon_{3}=0.2, \quad g_{1}=2.0, \quad g_{2}=2.0 ;$ and (b) velocity history of the Duffing equation under additive and multiplicative noises along with a periodic excitation; $\varepsilon_{1}=0.25, \quad \varepsilon_{2}=1.0$, $\varepsilon_{3}=0.2, \quad g_{1}=2.0, \quad g_{2}=2.0$.

these comparisons are shown when all the three different loads, i.e. periodic, additive and multiplicative, are present. While comparisons of histories generated by BLTL and HLTL methods are usually good, in Figure 3, the BLTL scheme may be seen to have erred to quite an extent when the intensity of the multiplicative noise is rather high.

In the second set of examples, a symmetrically non-linear two-degree-of-freedom (TDOF) oscillator under a couple of additive noises is numerically integrated using the transversal linearization procedures. The differential equations, in the four-dimensional state space, 

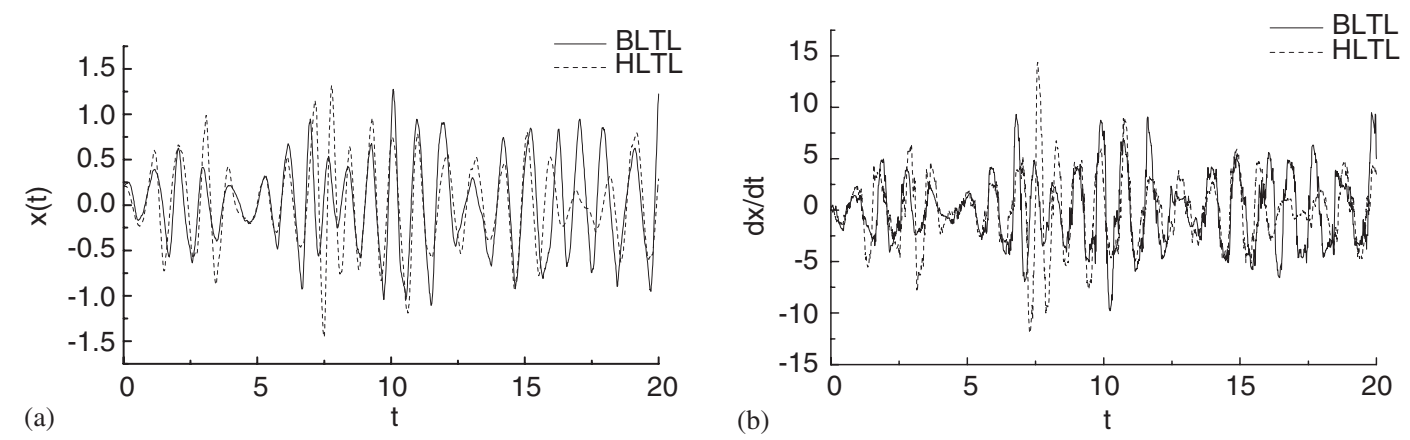

Figure 3. (a) Displacement history of the Duffing equation under additive and strong multiplicative noises along with a periodic excitation; $\varepsilon_{1}=0.25, \quad \varepsilon_{2}=1.0, \quad \varepsilon_{3}=0.2, \quad g_{1}=1.0, \quad g_{2}=10.0 ;$ and $(b)$ velocity history of the Duffing equation under additive and strong multiplicative noises along with a periodic excitation; $\varepsilon_{1}=0.25, \varepsilon_{2}=1.0$,

$$
\varepsilon_{3}=0.2, \quad g_{1}=1.0, \quad g_{2}=10.0 .
$$
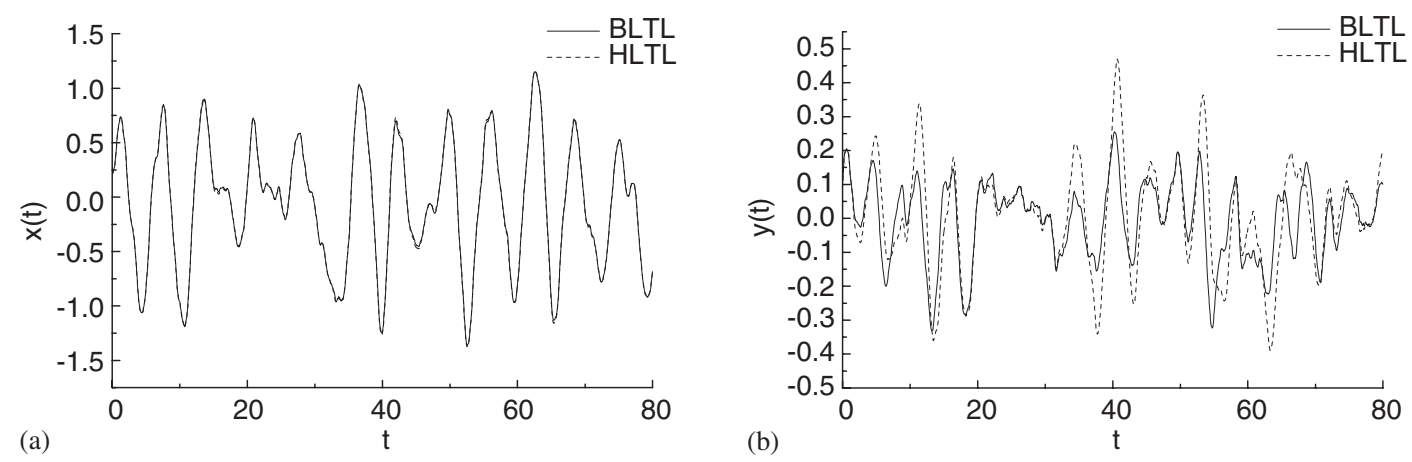

Figure 4. (a) History of $x(t)$ for a weakly non-linear TDOF equation under a couple of additive noises; $k_{1}=0.1, \quad k_{2}=0.3, k_{3}=0.3, k_{4}=0.1, g_{1}=0.5, g_{2}=0.2$; and (b) history of $y(t)$ for a weakly non-linear TDOF equation under a couple of additive noises; $k_{1}=0.1, k_{2}=0.3, k_{3}=0.3$,

$$
k_{4}=0.1, \quad g_{1}=0.5, g_{2}=0.2 \text {. }
$$

are presently given by

$$
\begin{aligned}
& \mathrm{d} x_{1}=x_{2} \mathrm{~d} t \\
& \mathrm{~d} x_{2}=\left(-0.4 x_{2}-x_{1}-k_{1} x_{1}^{3}-k_{2} x_{1} y_{1}^{2}\right) \mathrm{d} t+g_{1} \mathrm{~d} W_{1}(t) \\
& \mathrm{d} y_{1}=y_{2} \mathrm{~d} t \\
& \mathrm{~d} y_{2}=\left(-y_{2}-2 y_{1}-k_{3} x_{1}^{3}-k_{4} y_{1}^{3}\right) \mathrm{d} t+g_{2} \mathrm{~d} W_{2}(t)
\end{aligned}
$$

The steps for derivations of lower- and higher-order LTL systems for this TDOF problem remain the same as for the single DOF Duffing oscillator and are therefore not elaborated further. The following numerical results are obtained for two different sets of values of the 


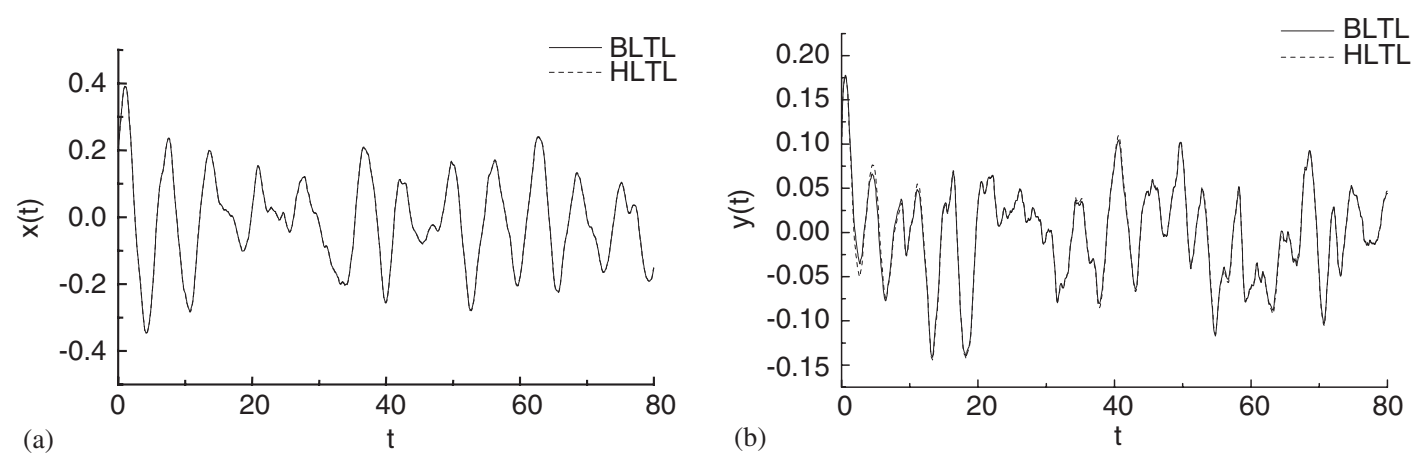

(a)

Figure 5. (a) History of $x(t)$ for a strongly non-linear TDOF equation under a couple of weak additive noises; $k_{1}=0.5, \quad k_{2}=0.6, \quad k_{3}=0.6, \quad k_{4}=0.3, \quad g_{1}=0.1, \quad g_{2}=0.1$; and (b) history of $y(t)$ for a strongly non-linear TDOF equation under a couple of weak additive noises; $k_{1}=0.5, k_{2}=0.6, k_{3}=0.6$, $k_{4}=0.3, \quad g_{1}=0.1, \quad g_{2}=0.1$.
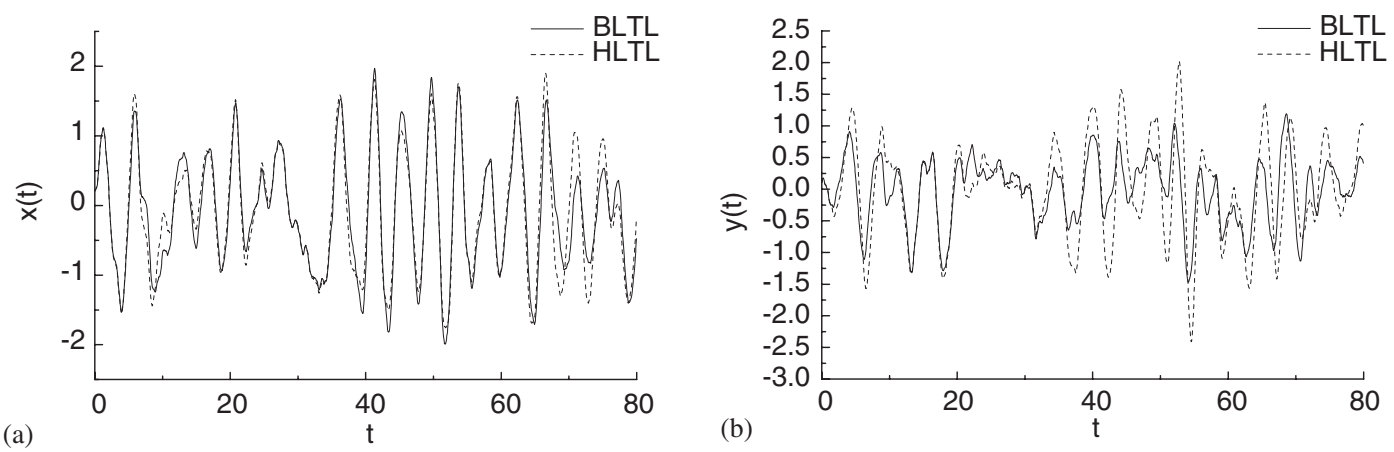

Figure 6. (a) History of $x(t)$ for a strongly non-linear TDOF equation under a couple of strong additive noises; $k_{1}=0.5, \quad k_{2}=0.6, \quad k_{3}=0.6, \quad k_{4}=0.3, \quad g_{1}=1.0, \quad g_{2}=1.0$; and (b) history of $y(t)$ for a strongly non-linear TDOF equation under a couple of strong additive noises; $k_{1}=0.5, k_{2}=0.6, k_{3}=0.6$,

$$
k_{4}=0.3, \quad g_{1}=1.0, \quad g_{2}=1.0 \text {. }
$$

parameters, namely the weakly non-linear case with $\left(k_{1}, k_{2}, k_{3}, k_{4}\right)=(0.1,0.3,0.3,0.1)$ and the strongly non-linear case with $\left(k_{1}, k_{2}, k_{3}, k_{4}\right)=(0.5,0.6,0.6,0.3)$. Figure 4 shows the histories for $x(t)$ and $y(t)$ for TDOF equation under a couple of additive noises of medium intensities (i.e. $g_{1}=0.5, g_{2}=0.2$ ) as obtained via both the BLTL and HLTL methods. In Figures 5 and 6 , the time histories of $x(t)$ and $y(t)$ for a strongly non-linear TDOF equation under weak and strong additive noise intensities are reported. While the comparisons of BLTL and HLTL solutions are generally good, they appear to differ to an extent in some cases, especially when the intensities of stochastic excitations are quite high. 
Finally it may be noted that comparisons of BLTL and HLTL solutions with those obtained with other popular schemes, especially the stochastic Heun or Euler schemes, are not provided here as the transversal linearization schemes have already been shown to have a higher accuracy over a given time step.

\section{CONCLUSIONS}

A new family of locally transversal linearization (LTL) procedures for efficient and accurate integration of non-linear stochastic engineering dynamical systems is proposed and theoretically explored for their local and global error orders. Detailed estimates for local and global error orders for displacement and velocity components are provided. These estimates are based on implicit Ito-Taylor expansions of these components in terms of the original and LTL-based vector fields. The LTL methodologies espoused in this study are broadly classified into two categories, namely the lower order or basic LTL (BLTL) method and the higher order LTL (HLTL) method. As their respective names suggest, the HLTL method is designed to improve upon the accuracy of the BLTL method, especially for systems under a sufficiently general set of multiplicative excitations. Even though the tool for solving the BLTL and HLTL systems remains essentially the same, solutions for the latter require a higher computational overhead owing to a higher dimensionality (1.5 times that of the corresponding BLTL system) of an HLTL system. In so far as the local error orders are concerned, the presently developed techniques are notably higher in accuracy than most other existing algorithms, such as the Heun scheme or similar other schemes based on stochastic Runge-Kutta. The only other competing algorithm, using a direct stochastic Taylor expansion of displacement and velocity vectors and leading (theoretically) to similar accuracy levels, involves extremely cumbersome (and, sometimes, nearly impossible) computations of multiple stochastic integrals. The BLTL and HLTL methodologies however effectively avoid such complexities. A limited numerical exploration of the proposed algorithms is provided by obtaining sample path solutions of a single-degree and a two-degree freedom, symmetrically non-linear dynamical systems under additive and multiplicative white noise excitations.

While the present study only deals with sample path integration strategies, approximating the moment functions, or more generally, the probability density or characteristic functions is also an important issue. Towards this, the author is presently in the final stages of developing a family of weak forms of the transversal linearization method. Efforts are also under way to see whether the LTL-based stochastic maps for engineering dynamical systems belong to a generalized symplectic form or not. The author hopes to report a few interesting results on these lines in a few forthcoming articles.

\section{ACKNOWLEDGEMENTS}

The author wishes to thank the referees for some their comments that have helped considerably in making the revised manuscript more readable.

\section{REFERENCES}

1. Gillespie DT. Markov Processes: An Introduction for Physical Scientists. Academic Press Inc: San Diego, CA, 1992.

2. Gard TC. Introduction to Stochastic Differential Equations. Marcel Dekker Inc: New York, 1988. 
3. Kloeden PE, Platen E. Numerical Solution of Stochastic Differential Equations. Springer: Berlin, 1999.

4. Maruyama G. Continuous Markov processes and stochastic equations. Rendiconti del Circolo Matematica di Palermo 1955; 4:48-90.

5. Gikhman II, Skorokhod AV. Stochastic Differential Equations. Springer: Berlin, 1972.

6. Kanagawa S. Confidence intervals of discretized Euler-Maruyama approximate solutions of SDEs. Nonlinear Analysis 1997; 30:4101-4104.

7. Wagner W, Platen E. Approximation of Ito integral equations. Preprint, ZIMM, Akad. Wissenschaften, DDR, Berlin, 1978.

8. Milstein GN. Numerical Integration of Stochastic Differential Equations. Kluwer Academic Publishers: Dordrecht, 1995.

9. Kloeden PE, Platen E. Higher order implicit strong numerical schemes for stochastic differential equations. Journal of Statistical Physics 1992; 66:283-314.

10. Rumelin W. Numerical treatment of stochastic differential equations. SIAM Journal on Numerical Analysis 1982; 19:604-613.

11. Roy D. A new numeric-analytic principle for non-linear deterministic and stochastic dynamical systems. Proceedings of the Royal Society of London A 2001; 457:539-566.

12. Leung AYT. Fast matrix exponent for deterministic or random excitations. International Journal for Numerical Methods in Engineering 2001; 50:377-394. 